\begin{abstract}
OPTIMIZATION OF THE MUON STOPPING TARGET FOR THE MU2E COLLABORATION

\author{
Zachary Donovan Hodge, M.S. \\ Department of Physics \\ David Hedin, Director
}

Northern Illinois University, 2013
\end{abstract}

Mu2e is a planned experiment to search for a flavor-violating conversion of a muon to an electron. The muon stopping target is a critical component of the experiment's hardware, having the task of stopping the muons and facilitating the rare conversion process. Incoming muons will interact with the aluminum stopping target and lose energy. Muons below a certain energy will come to rest in the $1 \mathrm{~S}$ orbital of an aluminum atom, where it then will either be captured by the nucleus, decay in orbit, or undergo a flavor-violating conversion to a monoenergetic electron (Mu2e signal). Improvements to the muon stopping target can lead to significant performance gains in the experiment overall.

Optimization of the stopping target has focused on increasing the number of muons that stop in target layers and improving track reconstruction efficiency by minimizing energy loss from nearby target layers. Several variations on target geometry, including mass distribution and spatial placement, have been conducted. A significant improvement in the physics capabilities of the Mu2e experiment has shown to be possible in this study. 
NORTHERN ILLINOIS UNIVERSITY

DE KALB, ILLINOIS

AUGUST 2013

\title{
OPTIMIZATION OF THE MUON STOPPING TARGET FOR THE MU2E COLLABORATION
}

\author{
BY \\ ZACHARY DONOVAN HODGE \\ (C) 2013 Zachary Donovan Hodge
}

A THESIS SUBMITTED TO THE GRADUATE SCHOOL

IN PARTIAL FULFILLMENT OF THE REQUIREMENTS

FOR THE DEGREE

MASTER OF SCIENCE

DEPARTMENT OF PHYSICS

Thesis Director:

David Hedin 


\section{ACKNOWLEDGEMENTS}

I would first like to thank both my mother and father for supporting and encouraging my interests in physics throughout my life. Without their guidance, my accomplishments would not be possible.

I would also like to thank my advisor, Dr. David Hedin, for his guidance through this master's, with endless insights and stories.

I would like to thank Dr. Robert Bernstein, Dr. Marjorie Corcoran, Dr. David Hitlin, Dr. Adam Yurkewic, and Dr. Avdhesh Chandra as well as all the members of the Mu2e Collaboration for their support while working on this project.

I want to extend my gratitude to the other members of my thesis committee, Dr. Mike Eads and Dr. Yasuo Ito, for their assistance during this thesis.

I would like to thank all the faculty, staff, and my fellow students in the NIU Physics Department.

I want to thank Megan Conway for her endless support.

I want to acknowledge the National Science Foundation for their funding and support. 


\section{DEDICATION}

To my family and friends 


\section{TABLE OF CONTENTS}

Page

LIST OF TABLES ............................. vi

LIST OF FIGURES. . . . . . . . . . . . . . . . . . . . . vii

Chapter

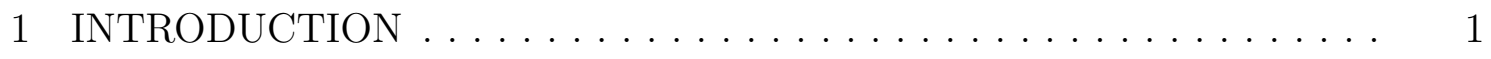

2 INTRODUCTION TO PARTICLE PHYSICS AND CHARGED LEP-

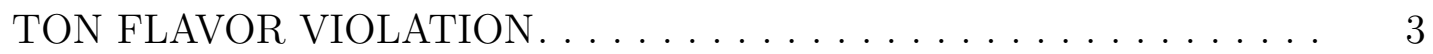

2.1 Introduction and Motivation . . . . . . . . . . . . . . 3

2.1 .1 Quark Mixing. ..................... 4

2.1 .2 Neutrino Oscillations . . . . . . . . . . . . 5

2.1 .3 Motivation for CLFV ................ 6

2.2 Current Status . . . . . . . . . . . . . . . . 7

2.2.1 Muon Flavor-Violating Channels . . . . . . . . . . . . . . 9

2.2.2 Previous Experiments and Results . . . . . . . . . . . . . 9

2.3 Mu2e Signal Process . . . . . . . . . . . . . . . . . . . . 10

$2.4 \quad$ Mu2e Background Processes. . . . . . . . . . . . . . . . . . . 11

2.4 .1 Decay In Orbit electrons . . . . . . . . . . . . . . . 12

2.4.2 Radiative Pion Capture on nucleus. . . . . . . . . . . . 13

2.4.3 Radiative Muon Capture on nucleus. . . . . . . . . . . . . . 13

3 OVERVIEW OF THE MU2E EXPERIMENT . . . . . . . . . . . . . . . . 15

3.1 FNAL Accelerator Faciilites . . . . . . . . . . . . . . . 15 
Chapter $\quad$ Page

3.2 Mu2e Solenoids. . . . . . . . . . . . . . . . . . . . . 17

3.2.1 The Production Target and Production Solenoid. . . . . . . 19

3.2 .2 The Transport Solenoid. . . . . . . . . . . . . . . . . 20

3.2 .3 The Detector Solenoid. . . . . . . . . . . . . . . . . . 20

3.3 The Mu2e Stopping Target . . . . . . . . . . . . . . . 23

3.3 .1 Choice of Material . . . . . . . . . . . . . . . 23

3.3.2 Energy Loss in the Target Layers. . . . . . . . . . . . . 25

4 THE MU2E OFFLINE SOFTWARE AND SIMULATIONS. . . . . . . . 27

4.1 Protons on target and muons downstream. . . . . . . . . 27

4.2 Muons stopping in the target layers . . . . . . . . . . . 28

4.3 Muon Conversion and background processes . . . . . . . . . . 32

4.4 Evaluating Geometry Changes . . . . . . . . . . . . . . 32

5 VARIATIONS OF THE TARGET CONFIGURATIONS . . . . . . . . . 35

5.1 Increasing number of target layers . . . . . . . . . . . . 36

5.2 The effects of layer radius tapering. . . . . . . . . . . . 37

5.3 Symmetric tapering. . . . . . . . . . . . . . . . . 41

5.4 Constant radius with decreasing thickness. . . . . . . . . . . . 50

5.5 Constant radius with constant thickness . . . . . . . . . . . . 53

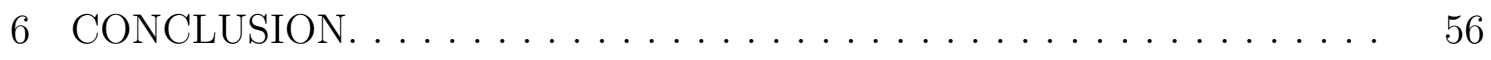

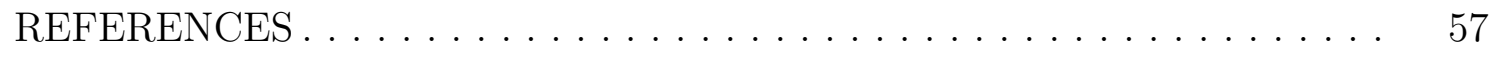




\section{LIST OF TABLES}

Table

Page

2.1 Fermions in the Standard Model . . . . . . . . . . . . . . . . 4

2.2 Current CLFV Branching Limits . . . . . . . . . . . . . . . . . . 10

4.1 Conversion electron track parameter cuts for yields. . . . . . . . . . 33

5.1 Radius of first and last foil for downstream tapering in $\mathrm{mm}$. . . . . 41

5.2 Radius of first and last foil for upstream tapering in $\mathrm{mm}$. . . . . . . 41 


\section{LIST OF FIGURES}

$\begin{array}{lll}\text { Figure } & \text { Page }\end{array}$

2.1 Muon flavor violation via $\mu-e$ conversion in the Standard Model. . 7

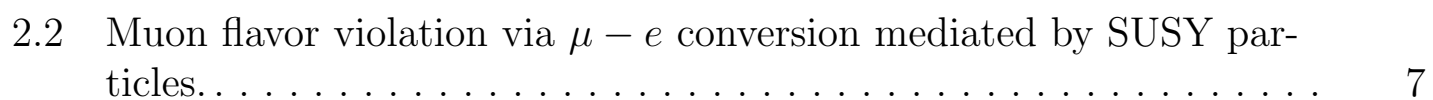

2.3 Lines represent branching ratios of different CLFV processes sensitive to new physics at energy scale $\Lambda$ as a function of $\kappa[7] . \ldots . .8$

2.4 The Michel spectrum of muons with a high side tail extending to

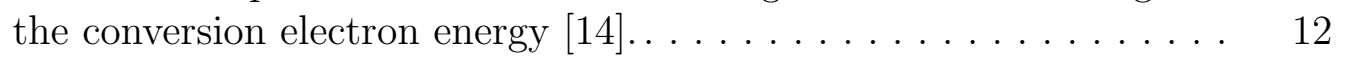

3.1 An overhead view of the FNAL accelerator complex. The Mu2e Experiment hall is located in the bottom right [14]. . . . . . . . 16

3.2 The proton bunch separation in time and search window with muon arrival time and muonic Al decay time overlaid [14]. . . . . . . . . . 17

3.3 An overhead view of the three solenoid sections of the Mu2e Exper-

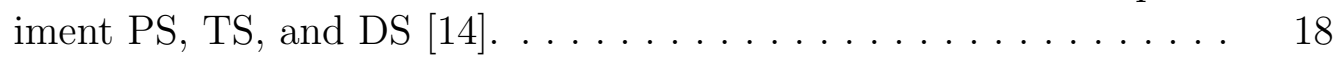

3.4 Cross-sectional view of the Production Solenoid [14]. . . . . . . . . . 19

3.5 Cross-sectional Top view of the Transport Solenoid. TS2 and TS4

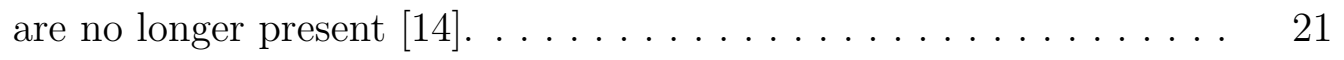

3.6 Cross-section top view of the Detector Solenoid with stopping target, tracker, calorimeter, and muon beam stop labeled[14]. . . . . . . 22

3.7 The standard Mu2e muon stopping target and tungsten support

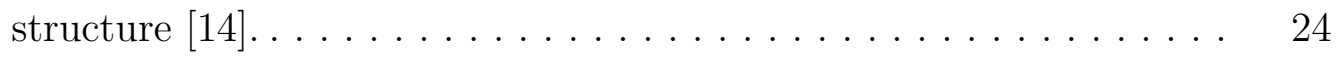

4.1 Incoming muon momentum distribution upstream of the first stop-

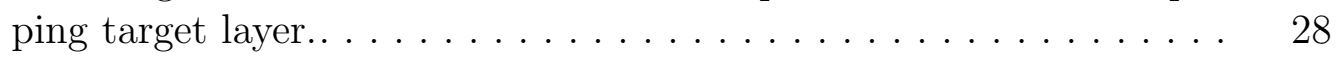

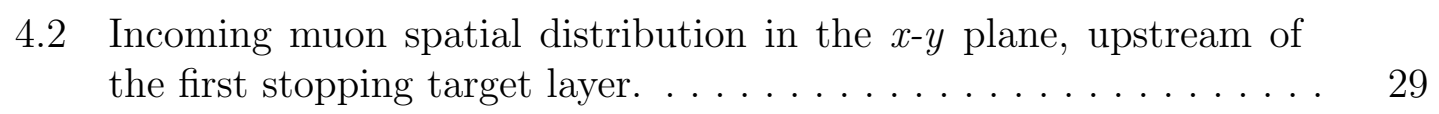


4.3 Distribution of incoming muon momentum as a function of radius, upstream of the first stopping target layer. . . . . . . . . . . 30

4.4 Stopped muon distribution in the target layers. . . . . . . . . . . . 31

$5.1 \quad$ Standard stopping target layer configuration for 17 foils. . . . . . . . 37

5.2 Stopping target layer configuration for 66 foils. . . . . . . . . . . 37

5.3 Yield versus Number of target layers from 13 to $90 . \ldots$. . . . . . . 38

$5.4 \quad 90 \%$ CL Sensitivity for increasing number of target layers. . . . . . . 39

5.5 Stopping target layer configuration for 66 foils with downstream

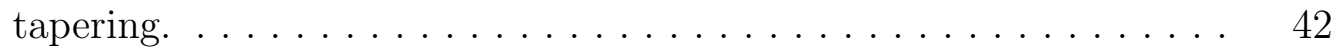

5.6 Stopping target layer configuration for 66 foils with upstream taper-

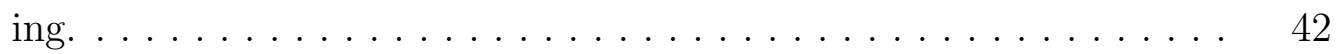

5.7 Yield versus foil radius for downstream tapering. . . . . . . . . . . . . 43

5.8 Yield versus foil radius for upstream tapering. . . . . . . . . . . . . . 44

$5.9 \frac{90 \% \text { CL sensitivity for all configuration of downstream tapering of }}{\text { target layer radius. } \ldots \ldots \ldots \ldots \ldots \ldots \ldots \ldots \ldots \ldots \ldots \ldots}$

$5.1090 \%$ CL sensitivity for all configuration of upstream tapering of tar-

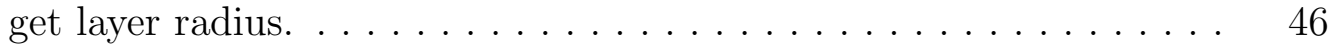

5.11 Stopping target layer configuration for 65 foils with symmetric ta-

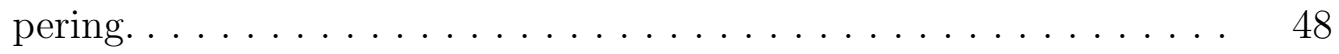

5.12 Yield versus foil radius for symmetric tapering. . . . . . . . . . . . . . 48

$5.1390 \%$ CL Sensitivity for all configuration of symmetric tapering of target layers. . . . . . . . . . . . . . . . . . . 49

5.14 Stopping target layer configuration for 66 foils with constant radius. 51

5.15 Yield versus foil radius for constant radius and constant mass. . . . . 51

$5.1690 \%$ CL sensitivity for all configuration of constant mass and increasing layer radius (decreasing thickness). . . . . . . . . . 52

5.17 Yield versus foil radius for constant radius and constant thickness. . 54 
$5.1890 \%$ CL Sensitivity for all configuration of constant thickness and increasing layer radius (increasing mass). . . . . . . . . . . . . 55 


\section{CHAPTER 1 INTRODUCTION}

Since the start-up of the Large Hadron Collider at CERN and the decommissioning of the Tevatron at Fermilab, the focus of American experimental particle physics has partially shifted to what is known as the Intensity Frontier [1]. This regime of physics experiments focuses on observing or setting new limits on very rare processes or exploring new fundamental interactions beyond the Standard Model. The Intensity Frontier consists of many sectors currently investigating rare processes and decays, such as

- Kaon and B meson decays

- Charged lepton flavor-violating processes

- Neutrino oscillations and mass measurements

- Proton, Neutron, and Electron electric dipole moments measurements

Investigating any rare process requires a large number of interactions, which are produced by high intensity beamlines, as well as measurement using very precise and sensitive detectors.

The charged lepton flavor-violation sector is one of many in the Intensity Fron-

tier program. Currently, there are several charged lepton flavor-violation (CLFV) experiments running or planned for the next decade. In particular to muon physics, the Mu2e Experiment at Fermilab will search for the flavor-violating neutrinoless conversion of a muon to electron. This process is predicted by several theories 
that include new physics and occur at rates within the expected capabilities of the experiment.

The Mu2e Experiment utilizes state of the art accelerators, superconducting magnets, detectors, electronics, and other equipment to maximize the sensitivity to such a rare process. Many of the components of the Mu2e hardware are critical to the overall physics capability of the experiment. The muon stopping target, where muons are stopped and may interact via this very rare process, is one such component where any improvements beyond the base design can have a significant impact on the experiment. This thesis explores possible modifications to the geometry of the muon stopping target. The goal is to determine if any modifications can improve the sensitivity of observing the muon conversion process. 


\section{CHAPTER 2}

\section{INTRODUCTION TO PARTICLE PHYSICS AND CHARGED LEPTON FLAVOR VIOLATION}

\subsection{Introduction and Motivation}

Currently, the Standard Model (SM) consists of three distinct sets of particles: gauge bosons, fermions, and the Higgs boson. The gauge bosons, $W^{ \pm}, Z^{0}, \gamma$, and gluons, carry integer spins and are the mediators of fundamental interactions. The fermions carry half integer spins and are organized into three generations as seen in Table 2.1. The Higgs boson explains the masses of the $W^{ \pm}$and $Z^{0}$ and generates the masses of the fermions.

The set of fermions can be further divided into quarks and leptons. The quarks carry both electric and color charges, and therefore participates in electromagnetic, weak, and strong interactions. Due to color confinement, a phenomenon that prevents colored particles from existing individually, quarks will form bound pairs with other quarks such that the net color charge is zero. These bound states are known as mesons (quark-antiquark pairs) and baryons (three quarks). The leptons consist of three charged particles and three corresponding neutral neutrinos. The three charged leptons participate in electromagnetic and weak interactions, whereas the neutrinos only participate in weak interactions [2]. 
Table 2.1: Fermions in the Standard Model

\begin{tabular}{|c|c|c|c|c|}
\hline & Electric Charge & 1st Gen & 2nd Gen & 3rd Gen \\
\hline \multirow{2}{*}{ Quarks } & $+2 / 3(-2 / 3)$ & $u(\bar{u})$ & $c(\bar{c})$ & $t(\bar{t})$ \\
\cline { 2 - 5 } & $-1 / 3(+1 / 3)$ & $d(\bar{d})$ & $s(\bar{s})$ & $b(\bar{b})$ \\
\hline \multirow{2}{*}{ Leptons } & $-1(+1)$ & $e^{-}\left(e^{+}\right)$ & $\mu^{-}\left(\mu^{+}\right)$ & $\tau^{-}\left(\tau^{+}\right)$ \\
\cline { 2 - 5 } & 0 & $\nu_{e}\left(\bar{\nu}_{e}\right)$ & $\nu_{\mu}\left(\bar{\nu}_{\mu}\right)$ & $\nu_{\tau}\left(\bar{\nu}_{\tau}\right)$ \\
\hline
\end{tabular}

\subsubsection{Quark Mixing}

The quark sector can undergo flavor-changing weak decays where the quark of one generation may decay or transition to another. In the case of quarks, loop level effects lead to observable quark generation transitions, such as $s \rightarrow d$. The observed suppression of flavor-changing neutral currents (FCNC) in $K^{0} \rightarrow \mu^{+} \mu^{-}$ was important in unveiling the existence of the charm quark through the GlashowIliopolous-Maiani (GIM) mechanism[3]. Later this was extended to predict a third generation of quarks. The weak mixing of the three quark generations is given by the Cabbibo-Kobayashi-Maskawa $(\mathrm{CKM})$ matrix $\mathbf{V}_{\mathbf{i j}}[2]$. The CKM matrix gives the probability of transition from quark flavor $\mathrm{i} \rightarrow \mathrm{j}$

$$
\left(\begin{array}{c}
\left|d^{\prime}\right\rangle \\
\left|s^{\prime}\right\rangle \\
\left|b^{\prime}\right\rangle
\end{array}\right)=\left(\begin{array}{ccc}
V_{u d} & V_{u s} & V_{u b} \\
V_{c d} & V_{c s} & V_{c b} \\
V_{t d} & V_{t s} & V_{t b}
\end{array}\right)\left(\begin{array}{c}
|d\rangle \\
|s\rangle \\
|b\rangle
\end{array}\right)
$$


Where $|q\rangle$ is the mass eigenstate and $\left|q^{\prime}\right\rangle$ is the up type quark flavor eigenstate which is a superposition of down types.

\subsubsection{Neutrino Oscillations}

In 1998 The Super-Kamiokande Collaboration showed that the atmospheric neutrino flux was inconsistent with that of flavor-conserving processes [4]. The mechanism proposed to explain this was called neutrino oscillation, whereby neutrinos produced of one flavor could oscillate and be measured as another flavor. The flavor eigenstates of neutrinos produced in weak interactions are related to the mass eigenstates via the lepton mixing matrix $\mathbf{U}_{\mathbf{i j}}$

$$
\begin{aligned}
& \left(\begin{array}{c}
\left|\nu_{e}\right\rangle \\
\left|\nu_{\mu}\right\rangle \\
\left|\nu_{\tau}\right\rangle
\end{array}\right)=U\left(\begin{array}{c}
\left|\nu_{1}\right\rangle \\
\left|\nu_{2}\right\rangle \\
\left|\nu_{2}\right\rangle
\end{array}\right) \\
& U=\left(\begin{array}{ccc}
-s_{12} c_{23}-c_{12} s_{23} s_{13} e^{\imath \delta} & c_{12} c_{23}-s_{12} s_{23} s_{13} e^{\imath \delta} & s_{23} \\
c_{12} c_{13} & s_{12} c_{13} & \\
s_{12} s_{23}-c_{12} c_{23} s_{13} e^{\imath \delta} & -c_{12} s_{23}-s_{12} c_{23} s_{13} e^{\imath \delta} & c_{23} c_{13}
\end{array}\right)
\end{aligned}
$$


where $c_{i j}=\cos \theta_{i j}$ and $s_{i j}=\sin \theta_{i j}$. This mixing arises from the non zero (though quite small) masses of the neutrino generations [5].

\subsubsection{Motivation for CLFV}

Given that in the fermion sector, both the quarks and neutrino experience some form of mixing, one would expect to see something similar in charged leptons. The Wienberg-Salam-Glashow (WSG) gauge theory of weak interactions only incorporates lepton flavor conservation with massless neutrinos. In fact, there is no global symmetry of the Lagrangian for which lepton number is universally conserved. It would seem more reasonable to expect that the suppression of CLFV channels comes from some dynamical mechanism [6]. In a modified SM for non-zero neutrino mass, CLFV is allowed. The rate at which CLFV is observed is highly dependent on the mechanism for neutrino mass generation, and generally unmeasurably small with simply non-zero neutrino mass. The SM branching ratio with massive neutrinos for $\mu \rightarrow e \gamma$ is given by

$$
B r(\mu \rightarrow e \gamma)=\frac{3 \alpha}{32 \pi}\left|\sum_{i=2,3} U_{\mu i}^{*} U_{e i} \frac{\Delta m_{1 i}^{2}}{M_{W}^{2}}\right|^{2}<10^{-54}
$$

where $\mathrm{U}$ is the lepton mixing matrix, $\Delta m_{1 i}^{2}$ is the neutrino mass-squared differences, and $M_{W}^{2}$ is the $W^{ \pm}$boson mass [7]. Some possible supersymmetric (SUSY) or grand unification extensions to SM predict additional particles that would mediate CLFV processes, and produce measurable branching ratios. Therefore any signal of CLFV would be a clear indication of new physics. The SUSY SU(5) branching ratio for $\mu^{+} \rightarrow e^{+} \gamma$ ranges from $10^{-15}$ to $10^{-13}$, which is in the range of current or planned experiments [8]. 


\subsection{Current Status}

There are several channels in which charged lepton flavor violation can manifest itself. Most simply, a higher generation lepton will decay to a lower generation without conserving flavor, such as $\mu \rightarrow e \gamma$. Another channel is the neutrinoless conversion of a muon to an electron in the field of atomic nucleus. Figure 2.1 shows the SM process of $\mu-e$ conversion and Figure 2.2 shows the SUSY mediated $\mu-e$ conversion process. The effective Lagrangian for CLFV in muon to electron conversion is given by the following:

$$
\mathcal{L}_{C L F V}=\frac{m_{\mu}}{(\kappa-1) \Lambda^{2}} \bar{\mu}_{R} \sigma_{\mu \nu} e_{L} F^{\mu \nu}+\frac{\kappa}{(1+\kappa) \Lambda^{2}} \bar{\mu}_{L} \gamma_{\mu} e_{L}\left(\sum_{q=u, d} \bar{q}_{L} \gamma^{\mu} q_{L}\right)
$$

where $\Lambda$ is the scale of new physics and $\kappa$ measures the new physics contribution to CLFV whether via some magnetic-moment type operator $(\kappa \gg 1)$ or a four-fermion interaction $(\kappa \ll 1)$ [7]. The energy scale that certain flavor-violating channels probe at given sensitivities as a function of $\kappa$ is shown in Figure 2.3.

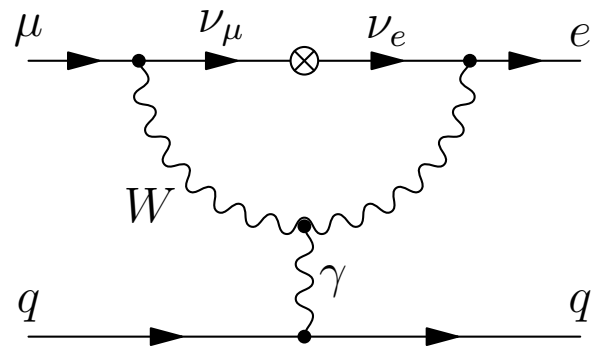

Figure 2.1: Muon flavor violation via $\mu-e$ conversion in the Standard Model.

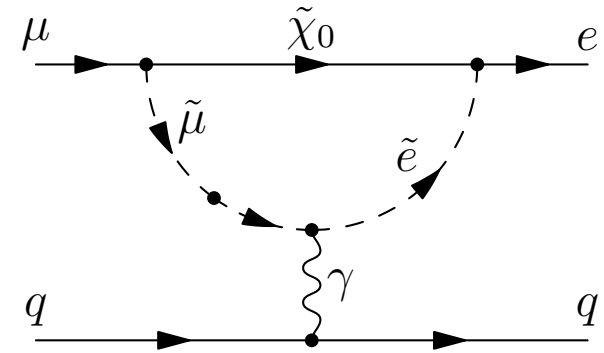

Figure 2.2: Muon flavor violation via $\mu-e$ conversion mediated by SUSY particles. 


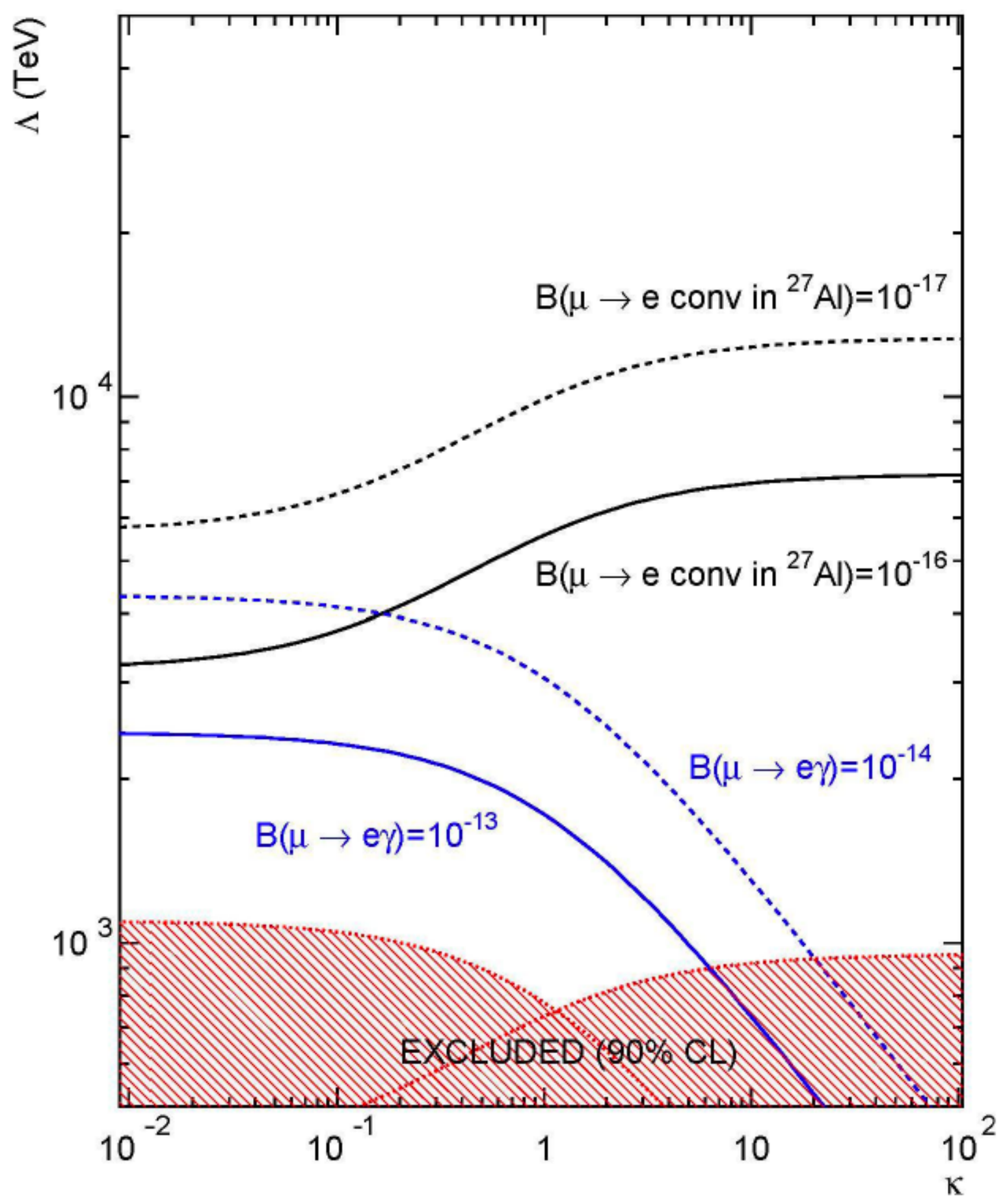

Figure 2.3: Lines represent branching ratios of different CLFV processes sensitive to new physics at energy scale $\Lambda$ as a function of $\kappa[7]$. 


\subsubsection{Muon Flavor-Violating Channels}

The flavor-violating channels in muons which are of most interest include $\mu \rightarrow$

$e \gamma, \mu \rightarrow e e e$, and $\mu+N \rightarrow e+N$. In general, the muons are stopped in some specified target material, which allows for them to either decay through SM processes or undergo a flavor-violating decay. The signal and prominent backgrounds are determined almost exclusively by the selection of stopping material. In the $\mu \rightarrow e \gamma$ process the electron and photon are emitted with energy equal to half the muon mass. The $\mu \rightarrow$ eee is similar to the previous, except that on off-shell photon produces an $e^{+} e^{-}$pair. The third channel, $\mu+N \rightarrow e+N$, involves the conversion of a muon to electron in the field of a nucleus. In a signal event, the final state electron is mono-energetic. Typical backgrounds in these channels include standard Michel decay, nuclear capture, radiative muon decay, and radiative pion capture [5].

\subsubsection{Previous Experiments and Results}

Several experiments have already been conducted on CLFV via muons. These types of experiments are primarily fixed target, where a produced muon beam is incident on some material that facilitates an interaction. Alternatively, CLFV can be probed in collider experiments such as Belle at KEK or BaBar at SLAC. The branching ratio limits set by these experiments can be found in Table 2.2. The TRIUMF experiment conducted several searches for $\mu-e$ conversion in various atomic nuclei [6].The SINDRUM II experiment was conducted at The Paul Scherrer Institut (PSI) in Switzerland looking for $\mu-e$ conversion in gold nuclei $\left(\mu^{-} A u \rightarrow\right.$ $\left.e^{-} A u\right)$ [9]. The MEG experiment, also conducted at PSI, is looking for $\mu^{+} \rightarrow e^{+} \gamma$ 
[10]. The MEGA experiment conducted at the Los Alamos National Laboratory Meson Physics Facility (LAMPF) searched for $\mu^{+} \rightarrow e^{+} \gamma[11]$. Searches for other LFV modes in tau decays were conducted by the BaBar and Belle experiments [12] [13].

Table 2.2: Current CLFV Branching Limits

\begin{tabular}{|c|c|c|}
\hline Process & Upper Limit & Experiment/Location \\
\hline$\mu^{-}+P b \rightarrow e^{-}+P b$ & $4.9 \times 10^{-10}$ & TRIUMF \\
\hline$\mu^{-}+A u \rightarrow e^{-}+A u$ & $7.0 \times 10^{-13}$ & SINDRUM II \\
\hline$\mu^{+} \rightarrow e^{+} \gamma$ & $1.2 \times 10^{-11}$ & MEGA at LAMPF \\
\hline$\mu^{+} \rightarrow e^{+} \gamma$ & $5.7 \times 10^{-13}$ & MEG at PSI \\
\hline \multirow{2}{*}{$\rightarrow \mu \gamma$} & $4.5 \times 10^{-8}$ & Belle \\
\cline { 2 - 3 } & $4.4 \times 10^{-8}$ & BaBar \\
\hline \multirow{2}{*}{$\tau \rightarrow e \gamma$} & $1.2 \times 10^{-7}$ & Belle \\
\cline { 2 - 3 } & $3.3 \times 10^{-8}$ & BaBar \\
\hline
\end{tabular}

\subsection{Mu2e Signal Process}

The Mu2e Experiment will measure the normalized relative conversion rate of muons to electrons, given by the following:

$$
R_{\mu e}=\frac{\Gamma\left(\mu^{-} N \rightarrow e^{-} N\right)}{\Gamma\left(\mu^{-} N \rightarrow \nu_{\mu} N^{\prime}\right)}
$$

The signal process for Mu2e is the neutrinoless conversion of muons to electrons in the field of Aluminum nuclei $\mu^{-} N \rightarrow e^{-} N$. Incoming muons with sufficiently low momentum will be stopped in the muon stopping target material, captured by the atomic nucleus, and cascade down to the $1 \mathrm{~S}$ state, forming a muonic atom where 
additional muon-nucleus interactions can take place. The conversion process results in a mono-energetic electron with energy $E_{\mu e}$ given in the following equation:

$$
E_{\mu e}=m_{\mu}-E_{b}-E_{r e c}
$$

where $m_{\mu}$ is the muon mass, $E_{b}=Z^{2} \alpha^{2} m_{\mu}$ is the muonic atom binding energy, and $E_{r e c}=m_{\mu}{ }^{2} / 2 m_{N}$ is the nuclear-recoil energy, $\alpha$ is the fine-structure constant, $m_{N}$ is the nucleus mass, and $Z$ is the atomic number of the stopping material. The Mu2e Experiment will use aluminum $(\mathrm{Z}=13)$ as the stopping target material, giving a conversion electron energy of $104.97 \mathrm{MeV}$. Due to energy loss and momentum resolution effects, the signal window defined by the Mu2e Experiment is a reconstructed electron with momentum $(p) 103.5<p<104.7 \mathrm{MeV}$ [14].

\subsection{Mu2e Background Processes}

There are several processes that can mimic the conversion signal and are collectively known as backgrounds. These backgrounds result from the primary muon beam or from particle interactions with material in the experiment. Backgrounds resulting from particle interactions with the stopping target are of primary concern. These background processes can occur during the detector data collection time and therefore must be accounted for and minimized. The estimation of background events can be found in Reference [15].The stopping target related background processes are described briefly in the following sections. All background estimates are given as the number of events in the signal window for a three-year run of data collection. 


\subsubsection{Decay In Orbit electrons}

The primary background process for Mu2e is muon decay in orbit (DIO), where a captured muon will decay to an electron and two neutrinos $\mu^{-} \rightarrow e^{-} \bar{\nu}_{e} \nu_{\mu}$ [16]. Due to this process occurring near the atomic nucleus, nuclear recoil effects can lead to the high energy tail of the Michel spectrum of the outgoing electron extending up to the conversion energy and is shown in Figure 2.4. The end point of this spectrum can be determined using numerical approximations found in Reference [16], and for aluminum is found to be $E_{\mu e}=104.973 \mathrm{MeV}$. The background due to DIOs is calculated to be $0.22 \pm 0.06$ events.

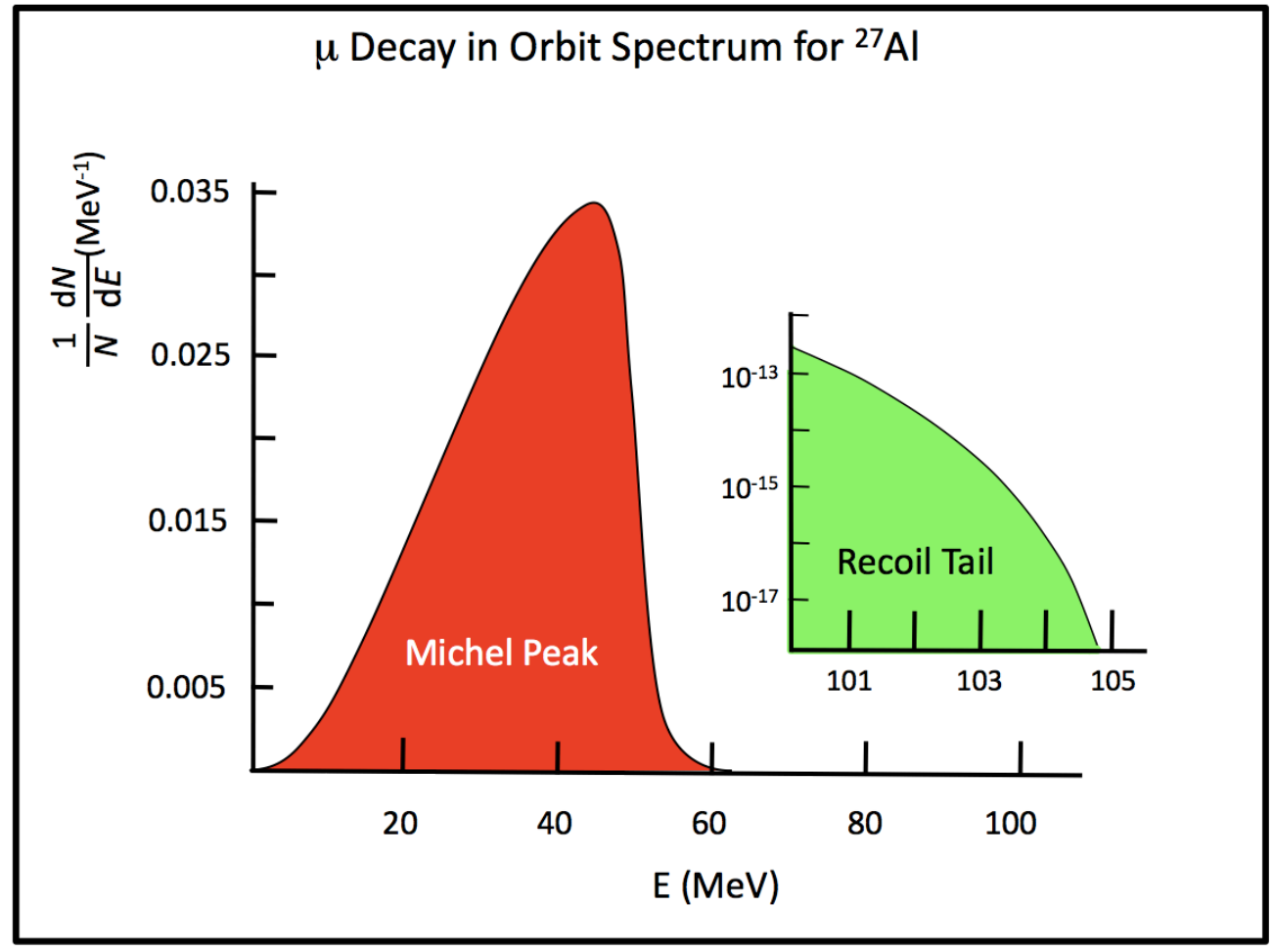

Figure 2.4: The Michel spectrum of muons with a high side tail extending to the conversion electron energy [14]. 


\subsubsection{Radiative Pion Capture on nucleus}

Pions that survive long enough to reach the Detector Solenoid can be stopped in the muon stopping target. These pions can produce background events through two nearly identical processes called radiative pion capture (RPC) [17]. First, a $\pi^{-}$ can undergo conversion to a photon through $\pi^{-} N(A, Z) \rightarrow \gamma N^{*}(a, Z-1)$, where the created photon subsequently produces an $e^{+} e^{-}$pair. Similarly, the photon can undergo internal conversion in the process $\pi^{-} N(A, Z) \rightarrow e^{+} e^{-} N^{*}(A, Z-1)$ at a rate about equal to external conversion. If the photon undergoes an asymmetric conversion, the electron will have enough energy to be in the signal region. Due to late arriving protons in the primary pulse, these pions can result in electrons being produced during the live gate time, leading to reconstruction of RPC electrons as conversion electrons. The background due to RPCs is estimated at $0.03 \pm 0.007$ events.

\subsubsection{Radiative Muon Capture on nucleus}

The final background process directly related to the muon stopping target is called radiative muon capture (RMC), where a muon is captured by the aluminum nucleus while radiating a photon giving the process $\mu^{-} A l \rightarrow \gamma \nu_{\mu} M g$ [18]. The outgoing photon can subsequently produce $e^{+} e^{-}$pairs. Similarly, the RMC photon can produce $e^{+} e^{-}$pairs through internal conversion at an equal rate. The endpoint energy can be determined by the following:

$$
k_{\text {max }}=m_{\mu} c^{2}-\Delta M-E_{r e c}-E_{b}
$$


where $m_{\mu}$ is the muon mass, $\Delta M$ is the nuclear mass difference, $E_{r e c}$ is the recoil energy, and $E_{b}$ is the binding energy. For aluminum, the endpoint energy is 102.4 $\mathrm{MeV}$. The background from RMC is estimated to be $<2 \times 10^{-6}$ events. 


\section{CHAPTER 3}

\section{OVERVIEW OF THE MU2E EXPERIMENT}

The Mu2e Experiment is currently in the advanced design phase at Fermi National Accelerator Laboratory (FNAL) in Batavia, IL USA. It is a fixed target experiment searching for the flavor-violating neutrinoless conversion of a muon to an electron in the field of an aluminum nucleus. The experiment relies heavily on existing beam lines and accelerator facilities at FNAL, with some modification and new beam lines required. The Mu2e Experiment is described in detail in Reference [14].

\subsection{FNAL Accelerator Faciilites}

At the FNAL accelerator complex, shown in Figure 3.1 a proton beam of $4 \times 10^{12}$ protons per batch is accelerated to $8 \mathrm{GeV}$ at the Fermilab Booster, which are then transported to the Recycler Ring via the MI-8 beam line. In the Recycler, the proton beam undergoes spatial and time structuring whereby each proton batch is converted into four bunches that are separated by 400 ns. From the Recycler, the protons are transported to the Debuncher ring, where protons are extracted from the beam with a 1695 ns peak-to-peak bunch separation, see Figure 3.2, and transported to the Mu2e production target. 


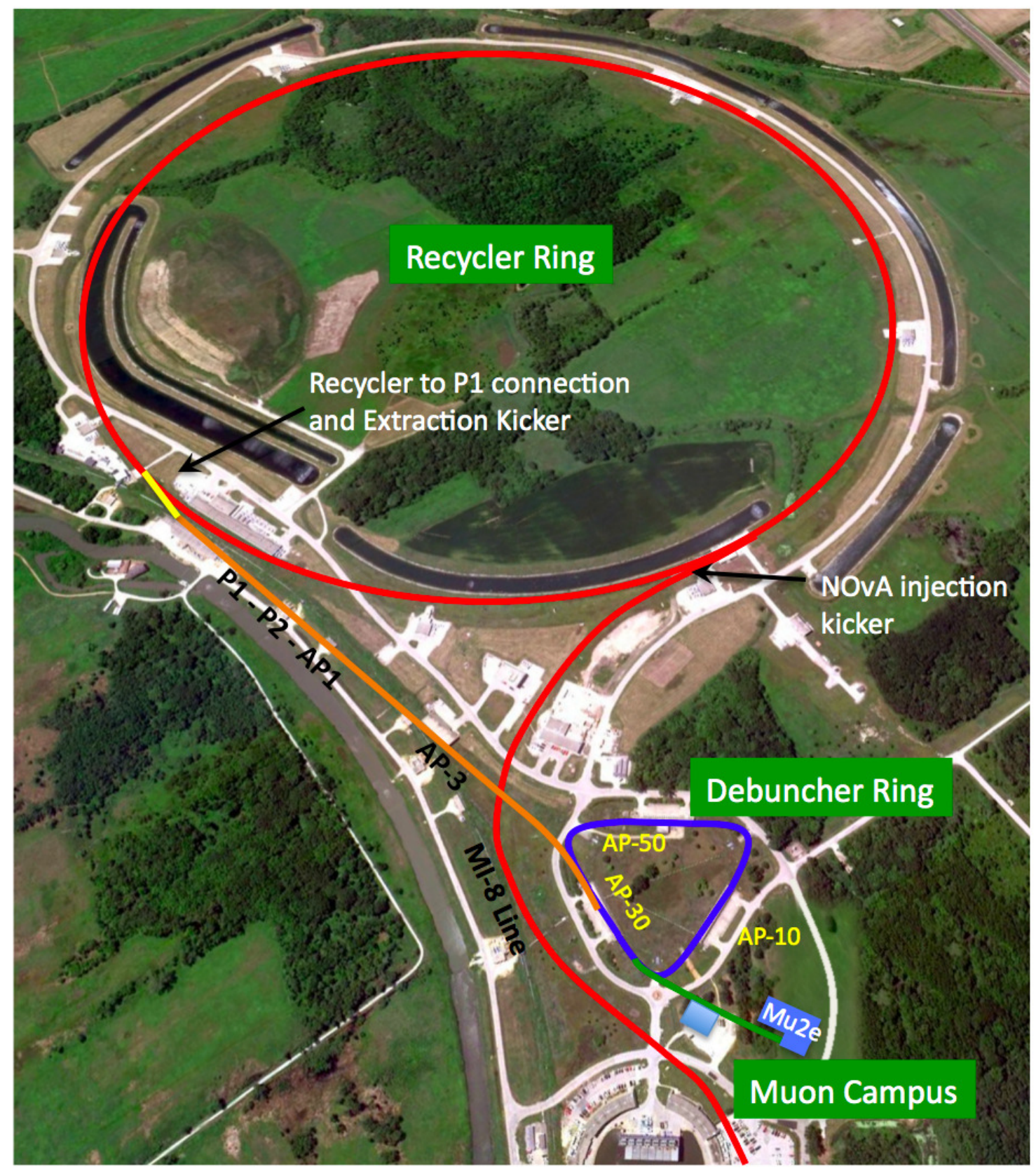

Figure 3.1: An overhead view of the FNAL accelerator complex. The Mu2e Experiment hall is located in the bottom right [14]. 


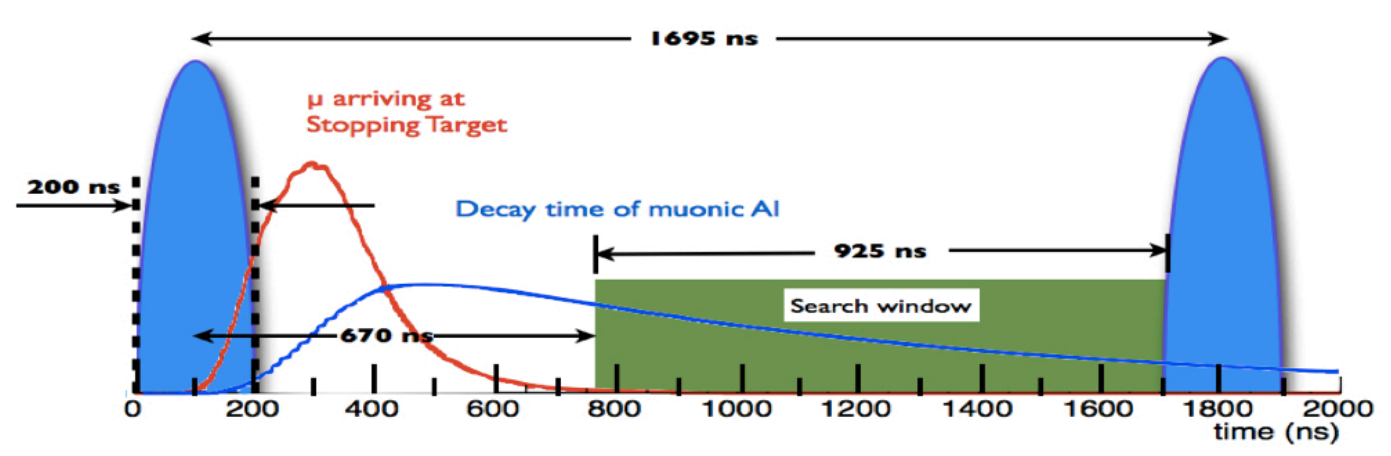

Figure 3.2: The proton bunch separation in time and search window with muon arrival time and muonic Al decay time overlaid [14].

\subsection{Mu2e Solenoids}

The Mu2e Experiment consists of three distinct sections of superconducting solenoids that facilitate the delivery of muons to the stopping target and passage of conversion electrons through the detector apparatus. The solenoids are designed to maximize the number of muons subsequently hitting the stopping target per primary proton on the production target. These solenoids are shown in Figure 3.3 and are labeled as the following: Production Solenoid (PS), Transport Solenoid (TS), and Detector Solenoid (DS).

There are three coordinate systems with different origins used by Mu2e [19]. The Mu2e coordinate system has an origin in the center of the TS with the $z$ axis parallel to the PS and DS solenoidal axes, the $y$ axis pointing vertically, and the $x$ pointing horizontally . The detector coordinate system has axes parallel to the Mu2e coordinate system with an origin in the center of the DS, located at (-3904.0, $0.0,12000) \mathrm{mm}$ in the Mu2e coordinate system. The tracker coordinate system 


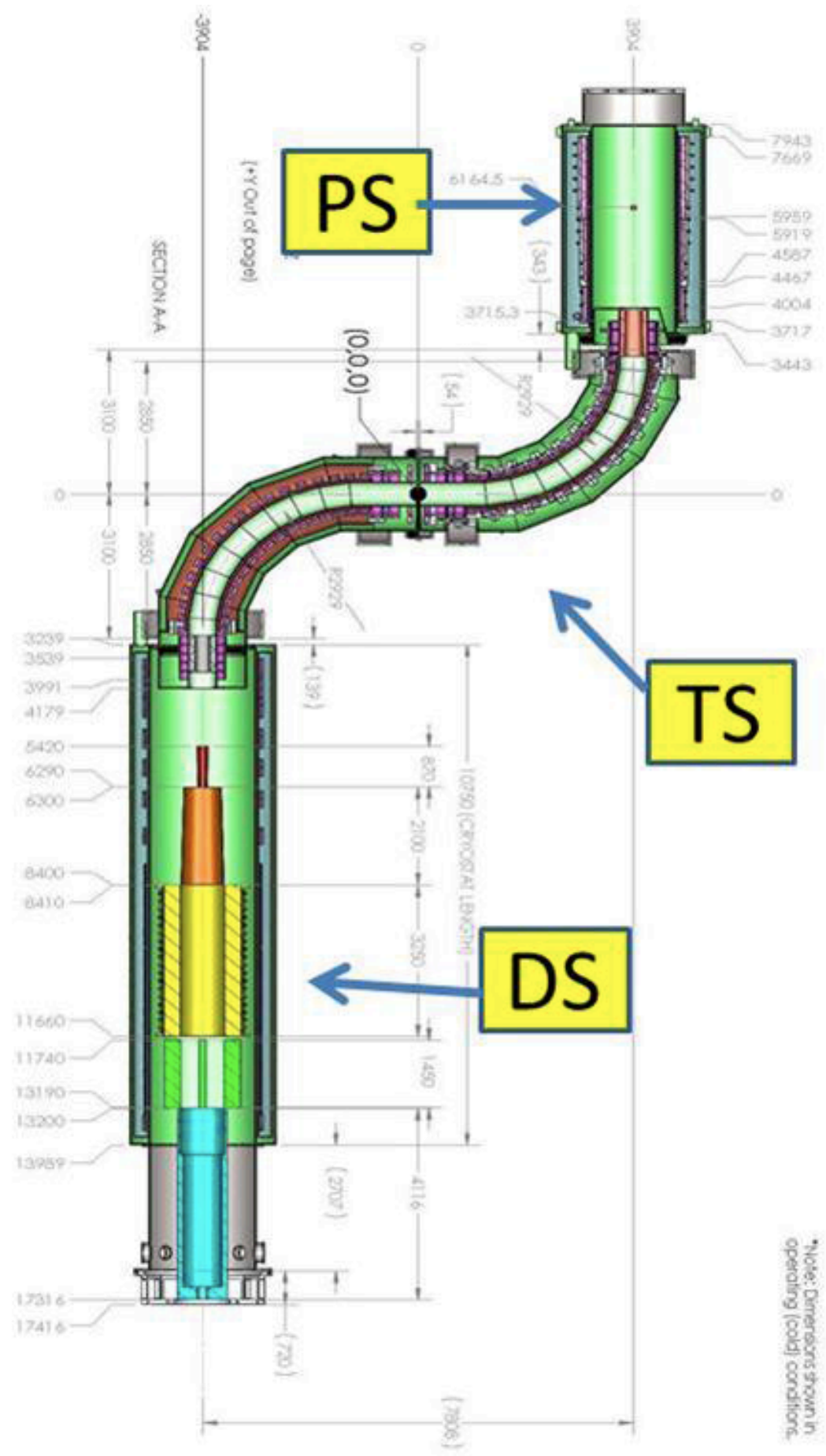

Figure 3.3: An overhead view of the three solenoid sections of the Mu2e Experiment PS, TS, and DS [14]. 
has axes parallel to the Mu2e coordinate system with an origin in the center of the tracker that is located at $(-3904.0,0.0,10200) \mathrm{mm}$ in the Mu2e coordinate system.

\subsubsection{The Production Target and Production Solenoid}

The incoming proton beam collides with a cylindrical tungsten target of 160 $\mathrm{mm}$ length and $3.15 \mathrm{~mm}$ radius, centered at (3904.0, 0.0, -6164.5) $\mathrm{mm}$ in the Mu2e coordinate system in the PS. See Figure 3.4 for a cross-sectional top view of the PS. The PS consists of an axially graded magnetic field that ranges from $4.6 \mathrm{~T}$ to 2.5 $\mathrm{T}$ which causes pions and other charged particles produced in the proton-target interaction to be accelerated toward the Transport Solenoid. Many of the pions will decay to muons in the PS and are pointed toward the TS. The expected production rate for Mu2e is 0.0016 stopped muons per proton on production target.

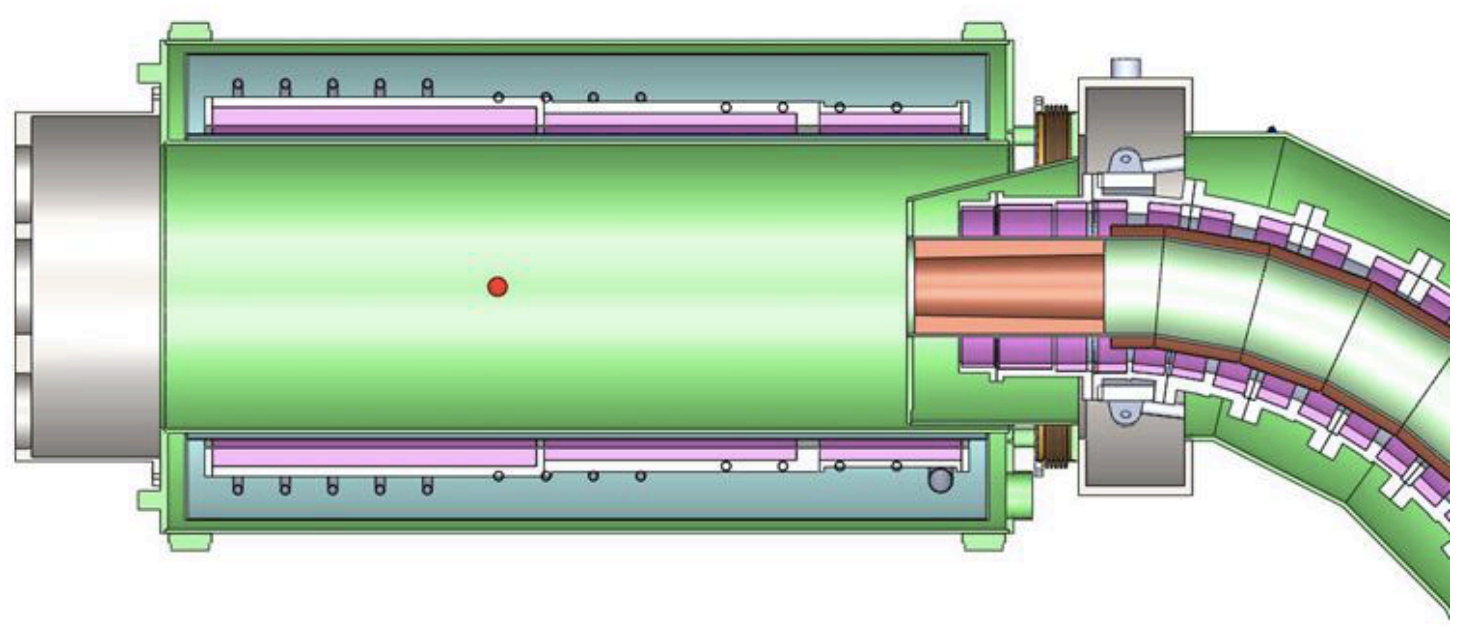

Figure 3.4: Cross-sectional view of the Production Solenoid [14]. 


\subsubsection{The Transport Solenoid}

The TS primary roles are to carry muons from the production solenoid to the Detector Solenoid, such that interactions relevant to the experiment occur near the detector hardware, and to passively minimize uninteresting particles from entering the detector volume. The TS consists of many sets of superconducting solenoids

and toroids that form a reverse $S$ shape. See Figure 3.5 for a overhead view of the TS.

The initial turn in the TS act to select against high energy charged particles and neutral particles. Inside the TS there are three collimators and absorbers (TS1, TS3, TS5) that act to reject positively charged particles from entering the DS. Figure 3.5 shows a top view of the TS and in the most recent design of the Transport Solenoid, the TS2 and TS4 collimators are no longer present.

\subsubsection{The Detector Solenoid}

A relatively low energy muon beam will enter the DS after being transported through the TS. The DS consists of both graded and uniform magnetic field sections. Figure 3.6 shows an overhead view of the PS, including the TS - DS interface. A graded field exists across the stopping target that ranges from $2 \mathrm{~T}$ to $1 \mathrm{~T}$. This field tends to pitch forward (toward the tracker) particles created in the stopping target with downstream momentum and reflect particles that are created with upstream momentum. Downstream of the stopping target resides the cylindrical shell polyethylene proton absorber, that acts to reduce the rates of protons entering the detector region. 


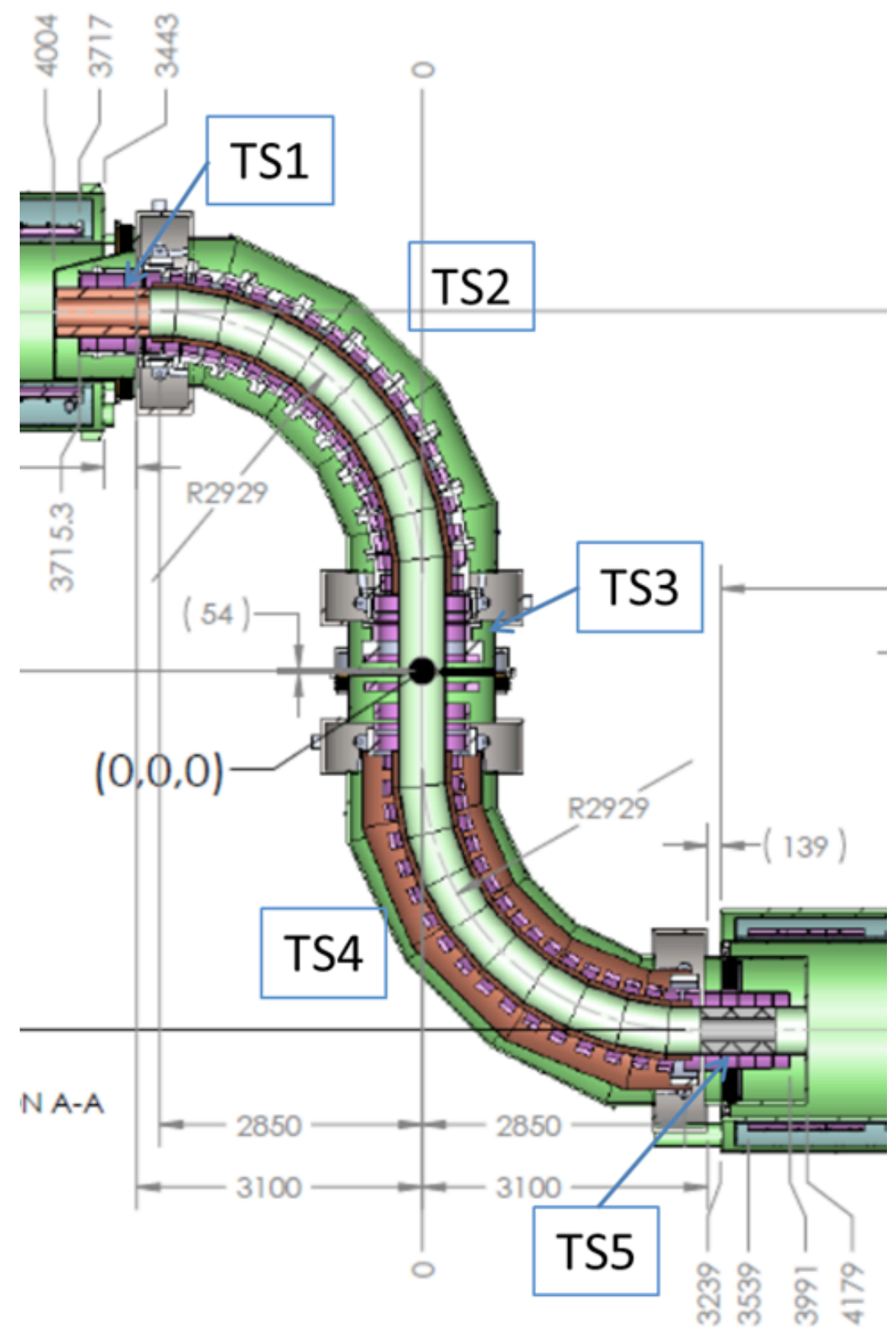

Figure 3.5: Cross-sectional Top view of the Transport Solenoid. TS2 and TS4 are no longer present [14. 


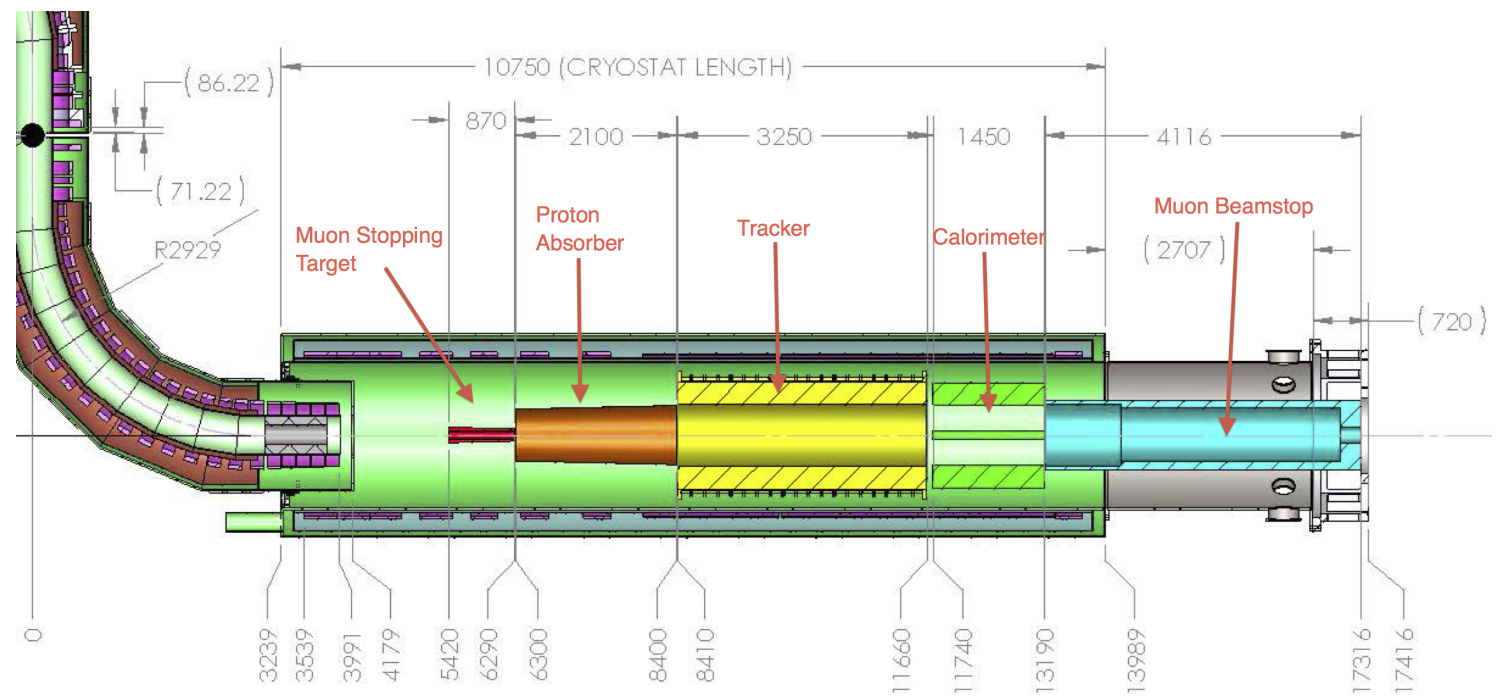

Figure 3.6: Cross-section top view of the Detector Solenoid with stopping target, tracker, calorimeter, and muon beam stop labeled[14].

The Mu2e detector consists of the tracker and calorimeter components, and reside in a region of uniform field. The tracker is a system of 22000 drift tubes aligned in the $x-y$ plane of the DS coordinate system. The tracker measures the momentum of electrons passing through the drift tubes and is optimized to distinguish between conversion electrons and DIO electrons. The calorimeter is a system of scintillating crystals and photodetectors designed to provide additional information such as energy, position, and timing of particles leaving tracks in the tracker. Farther downstream from the calorimeter resides the muon beam stop. The muon beam stop exists to capture remnant muons not stopped in the target, and shield detector components from particles produced in those muon captures. 


\subsection{The Mu2e Stopping Target}

The standard configuration the Mu2e muon stopping target is seventeen aluminum foil layers centered at $(0.0,0.0,-6129.0 \mathrm{~mm})$, ranging from $z=-6529 \mathrm{~mm}$ to $z=-5729 \mathrm{~mm}$ in the detector coordinate system. Each layer of the muon stopping target is separated from adjacent layers by $50 \mathrm{~mm}$. The radius of each layer decreases in the downstream direction, beginning with $83 \mathrm{~mm}$ and ending at $65.3 \mathrm{~mm}$. This decrease in radius is described as foil radius tapering. The thickness of each layer is the same for all layers and is $0.2 \mathrm{~mm}$. The density of aluminum is $2.70 \frac{\mathrm{g}}{\mathrm{cm}^{2}}$, and therefore the total mass of the muon stopping target is $159.4 \mathrm{~g}$.

The target is designed such that incoming muons will interact with enough mass to be stopped in the target volume and simultaneously each layer is thin enough to minimize the energy loss in conversion electron such that they will pass through the tracker and be properly reconstructed. The muon stopping target is supported by a structure consisting thin tungsten wires attached to each layer such that the layer is firmly suspended and centered on the incoming muon beam. Figure 3.7 shows the layers of the muon stopping target as well as the tungsten support structure.

\subsubsection{Choice of Material}

The material chosen for the muon stopping target is aluminum at $99.99 \%$ purity. There are several motivations for choosing aluminum, that include: muon conversion rate, muon lifetime, conversion electron energy, and related backgrounds. The stopped muon lifetimes decrease with increasing atomic number $(\mathrm{Z})$. The muon conversion rate increases with increasing $\mathrm{Z}$, with maximums at selenium and antimony, 


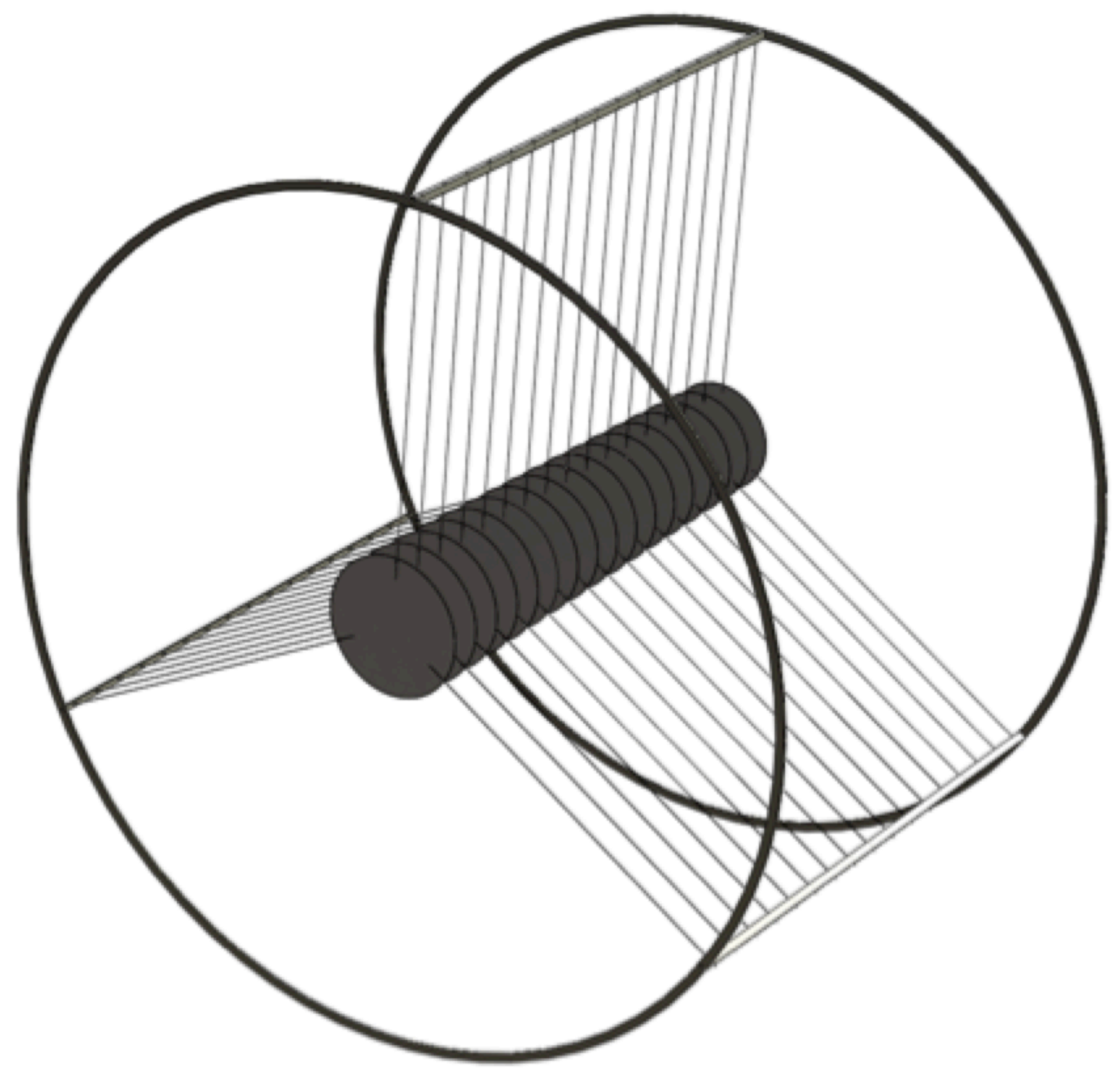

Figure 3.7: The standard Mu2e muon stopping target and tungsten support structure [14]. 
then decreases with increasing Z. For aluminum, the stopped muon lifetime is 864 ns and the $\mu-e$ conversion energy is $104.97 \mathrm{MeV}$. This relatively long muon lifetime is approximately half the time between proton bunches. This reduces the number of background events directly related to the muon beam occurring during the detector live gate time when the conversion electron process takes place.

\subsubsection{Energy Loss in the Target Layers}

The magnetic field found in the TS and DS causes charged particles to travel in helical paths as given by

$$
R=\frac{p_{T}}{q B}
$$

where $R$ is the radius of the helix, $p_{T}$ is the transverse momentum, $\mathrm{q}$ is the charge, and $\mathrm{B}$ is the magnetic field. When a charged particle interacts with matter it will lose energy in accordance with the Bethe-Bloch equation [20].

$$
\frac{d E}{d x}=\rho K z^{2} \frac{Z}{A} \frac{1}{\beta^{2}}\left[\frac{1}{2} \ln \left(\frac{2 m_{e} c^{2} \beta^{2} \gamma^{2} T_{\max }}{I^{2}}\right)-\beta^{2}\right]
$$

where $K=4 \pi N_{A} r_{e}{ }^{2} m_{e} c^{2}=0.307 M e V, r_{e}$ is the classical electron radius, $N_{A}$ is Avogadro's number, $m_{e}$ is the electron mass, $Z$ is the atomic number of the material, $A$ is the atomic mass number of the material, $I$ is the mean excitation energy of the material, $\rho$ is the density of the material, and $z$ is the incident particle's charge. The maximum kinetic energy that can be imparted to a free election in a collision is known as $T_{\max }$ and is given by

$$
T_{\max }=\frac{2 m_{e} c^{2} \beta^{2} \gamma^{2}}{1+\frac{2 \gamma m_{e}}{M}+\left(\frac{m_{e}}{M}\right)^{2}}
$$


As the muons in the Mu2e beam line traverse the stopping target volume, they will lose energy but due to their helical trajectory it is not guaranteed to cross the plane of every layer. Therefore determining exactly how much energy will be lost by a muon in the stopping target is difficult to predict. 


\section{CHAPTER 4}

\section{THE MU2E OFFLINE SOFTWARE AND SIMULATIONS}

The Mu2e Collaboration relies on several simulation software packages to design and optimize the components of the experiment. In particular, the Mu2e simulation software, also referred to as the framework, is built on the GEANT4 solid geometry and physics interaction software library [21]. Using this framework, all components of the Mu2e experiment can be constructed in the simulation environment. Within the framework, particle interactions with matter are simulated, and track reconstruction is conducted. In this study, all simulations were conducted using Mu2e "Offline v2_1_2".

\subsection{Protons on target and muons downstream}

Prior to the stopping target study, a simulation is performed that begins with the main $8 \mathrm{GeV}$ proton pulse. These protons collide with the production target, creating pions, which then subsequently decay to muons. These muons are carried through the TS to the DS. Inside the DS at $(0.0,0.0,-6539.5) \mathrm{mm}$ in the detector coordinate system, each individual muon's momentum, position, and proper time is saved to a file. The muons have an average momentum of $42 \mathrm{MeV} / \mathrm{c}$, with a high side tail extending up to $100 \mathrm{MeV} / \mathrm{c}$. The muon momentum distribution is shown in Figure 4.1. The incoming muon transverse position distribution is shown in Figure 4.2. The muon momentum versus radius from the solenoidal axis is shown in 
Figure 4.3. For perspective, downstream is defined as in the direction of the muon beam, from the TS-DS interface toward the tracker and calorimeter. Upstream is defined as the direction opposite of the muon beam and from the tracker and calorimeter toward the TS-DS interface.

\section{Incoming Muon Momentum}

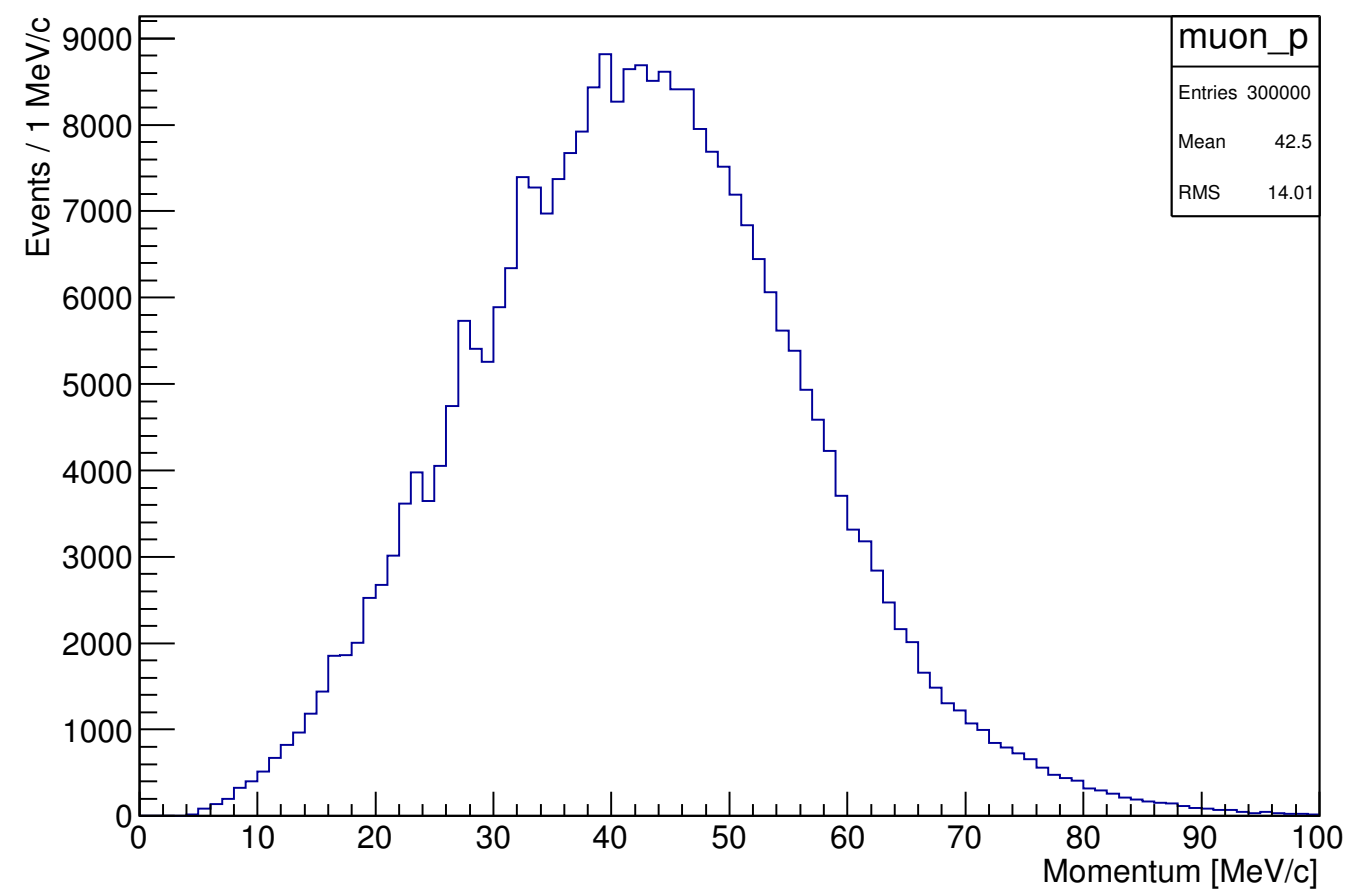

Figure 4.1: Incoming muon momentum distribution upstream of the first stopping target layer.

\subsection{Muons stopping in the target layers}

The muon file that was created from the initial simulation is then used as the input for all stopping target configurations. The first 300,000 muons are allowed to travel downstream through the DS where they interact with the muon stopping 


\section{Incoming Muons $\mathrm{Y}$ vs $\mathrm{X}$}

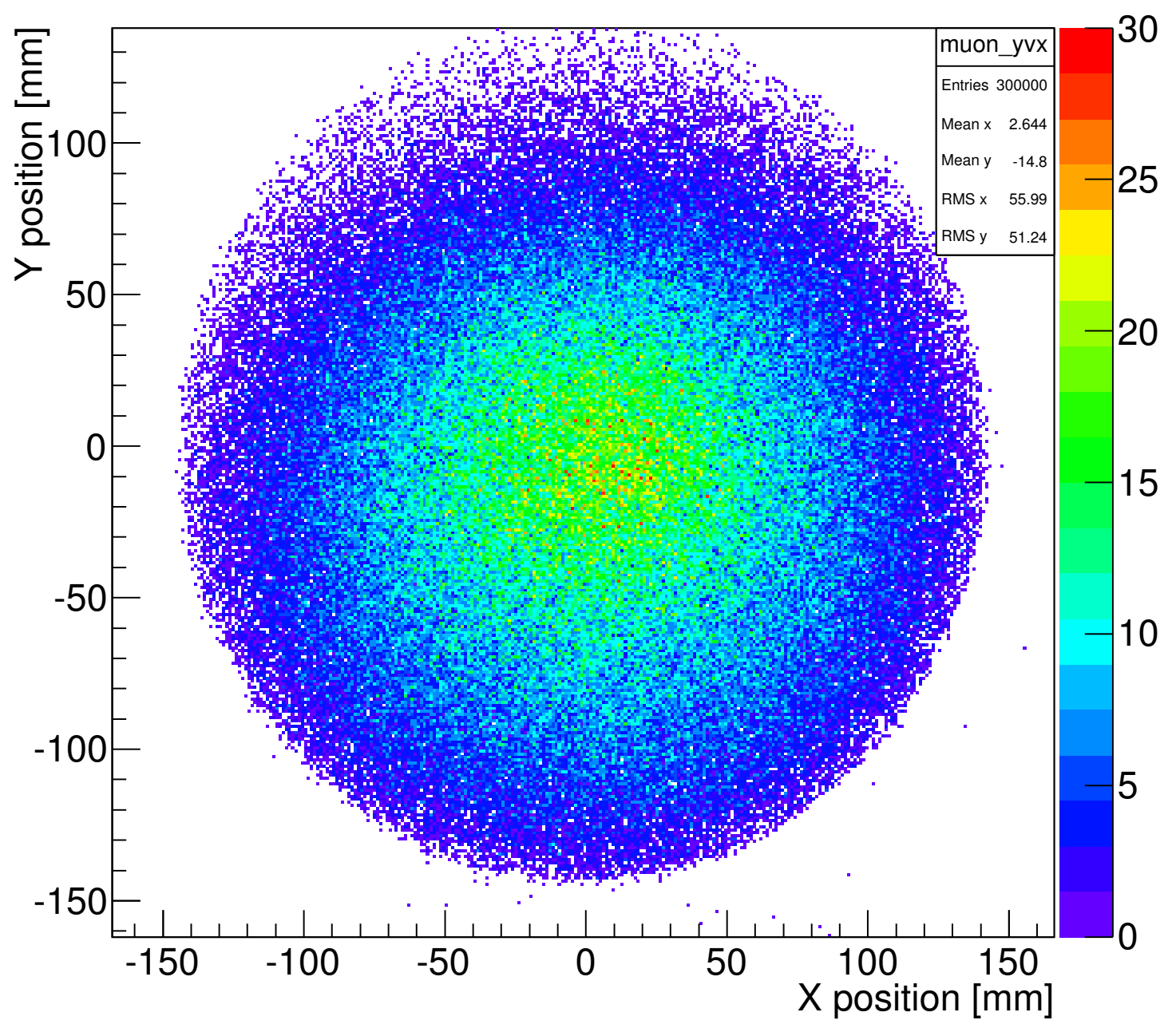

Figure 4.2: Incoming muon spatial distribution in the $x-y$ plane, upstream of the first stopping target layer. 


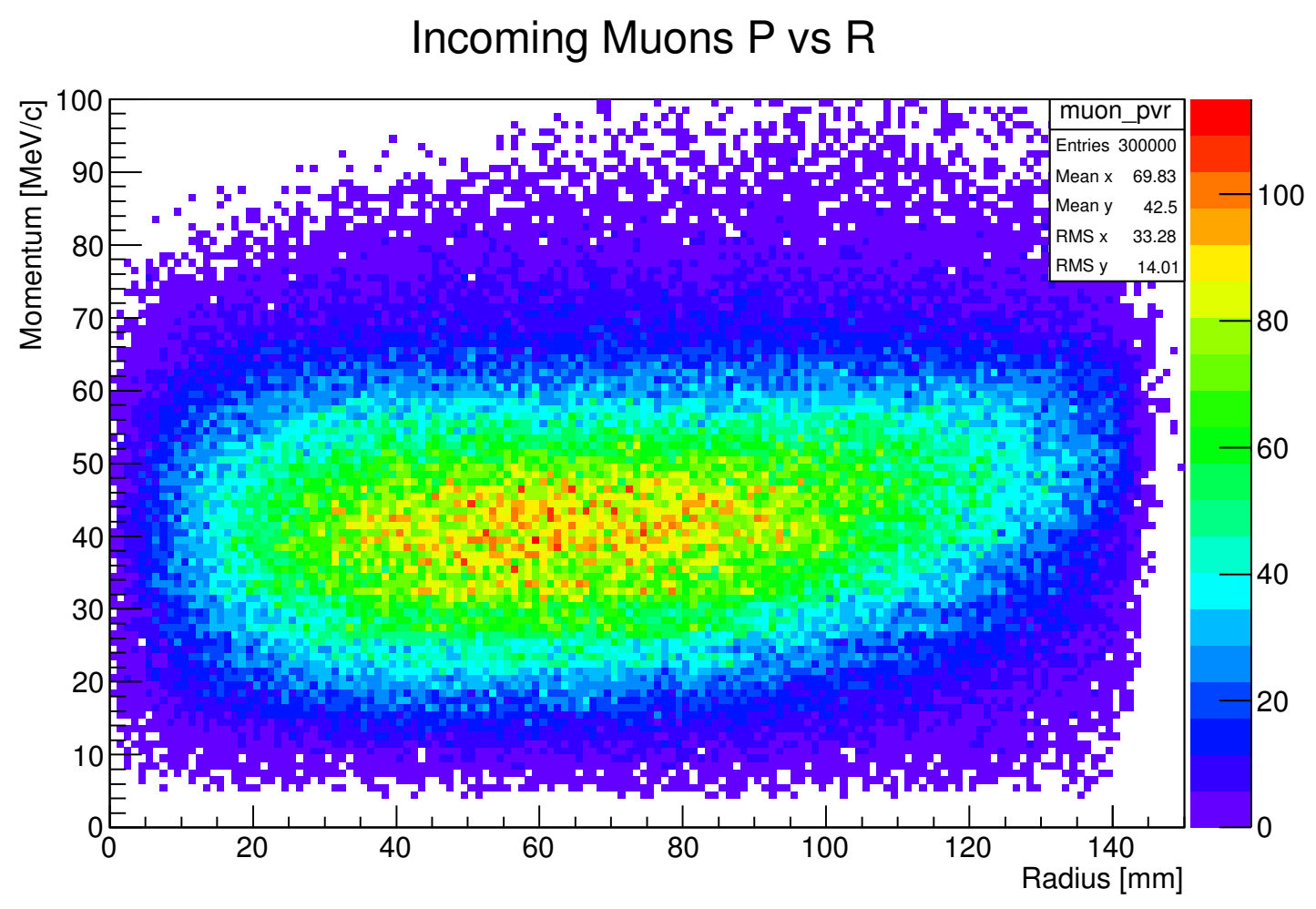

Figure 4.3: Distribution of incoming muon momentum as a function of radius, upstream of the first stopping target layer. 
target. In the standard target configuration approximately $47 \%$ of the muons will lose enough energy to be stopped by the muon stopping target.

Using the Particle Data Group table of muon energy loss in aluminum [22], one finds a muon beam with $42 \mathrm{MeV} / \mathrm{c}$ to have a range of $2.2 \mathrm{~mm}$ in Aluminum. The total thickness of the stopping target is $3.4 \mathrm{~mm}$, and using PDG table one would expect this amount of material to stop a muon of $100 \mathrm{MeV} / \mathrm{c}$ or less. However, due to the helical trajectory and part of the muon beam extending beyond the radius of the target, not all muons will strike all layers of the target or interact with the target at all. The muons that are stopped have their position and proper time saved to a file. The distribution of stopped muons in the target layers radius versus $\mathrm{Z}$ for the standard target configuration is shown in Figure 4.4.

Stopped Muons R vs Z

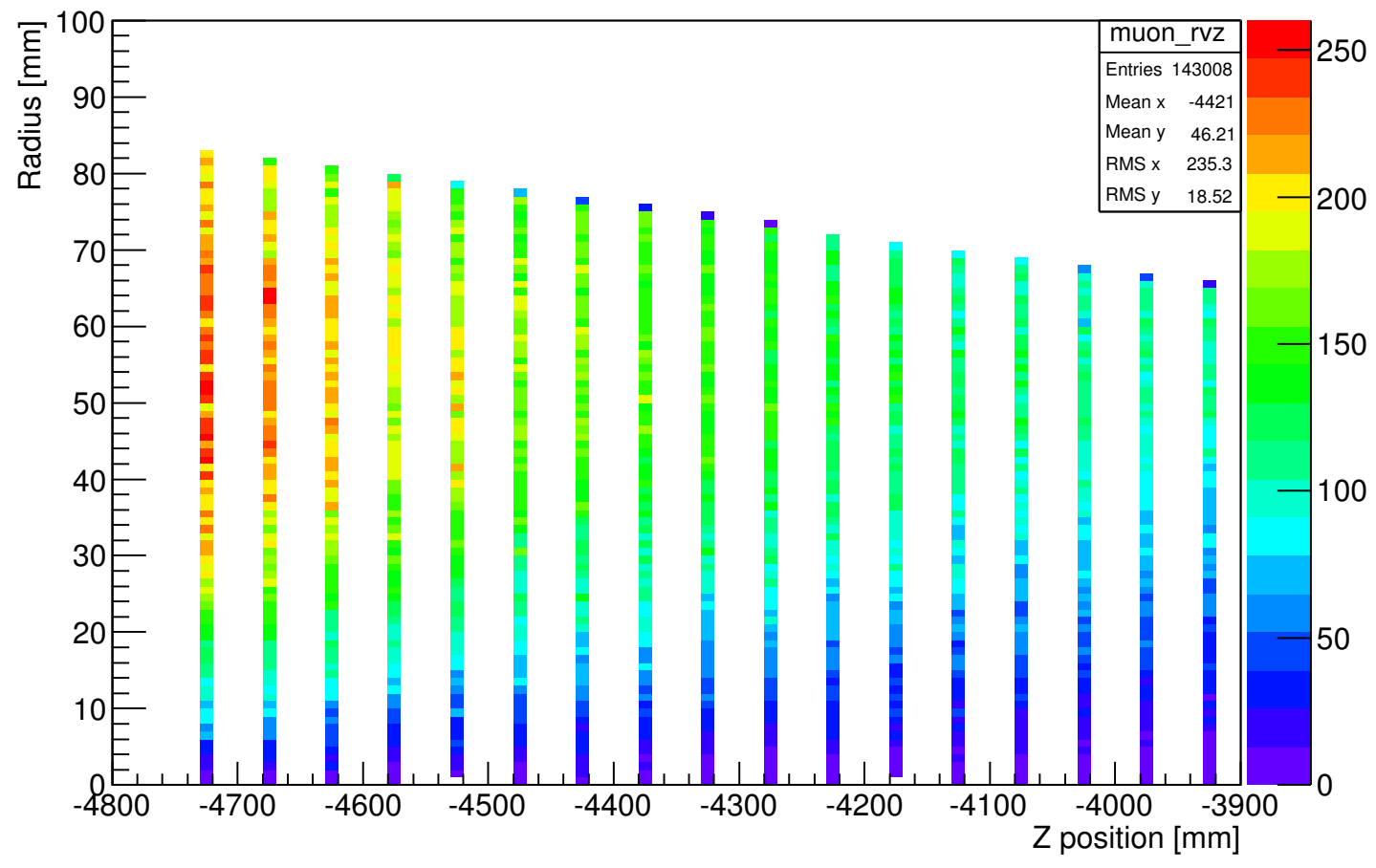

Figure 4.4: Stopped muon distribution in the target layers. 


\subsection{Muon Conversion and background processes}

The muons that have been stopped in the muon stopping target are then used as the input for subsequent simulation, where one of two possible scenarios occur. For the first, all the stopped muons are allowed to undergo the flavor-violation conversion to an electron. This is accomplished by "turning on" a framework generator process in the configuration file for the simulation. The process conversion process will force every stopped muon in the input file to undergo the flavor-violating neutrinoless conversion and create a mono-energetic electron.

Secondly, background process events must be generated for every target configuration. The most prominent background is electrons resulting from muon decay in orbit. To simulate DIO events, the DIO generator process is "turned on" which forces all stopped muons to undergo the decay in orbit background process. For all studies, the lower energy of the DIO spectrum is set to $101.5 \mathrm{MeV}$. This creates more DIO background events near the signal window, allowing to better study the effects of changing the muon stopping target geometry.

\subsection{Evaluating Geometry Changes}

This study will vary the stopping target geometry and see if improvements in the performance of Mu2e can be obtained. Ultimately, we want to increase the signal yield and/or reduce background rates, for example maximizing a figure of merit

such as the signal over root background $(S / \sqrt{B})$ for energies above $103.5 \mathrm{MeV}$. Two different techniques were used in this study. 
Initially, a criterion of improvement was established that would determine whether one target geometry configuration performed better than another. By taking all signal events created in a simulation, cuts were made against a list of reconstructed track components. A list of cuts used in this analysis can be found in Table 4.1. The total number of reconstruction events passing all these cuts constituted the signal events that would be observed by the experiment. This number is known as the "yield" and we abbreviate by "CE". Comparing yields from two or more target geometries allowed a determination of whether one configuration performed better than another.

Table 4.1: Conversion electron track parameter cuts for yields.

\begin{tabular}{|l|c|}
\hline Track parameter and cut & Description \\
\hline fitstatus $>0$ & returns 0 for unsuccessful track fit \\
\hline fitmom $<110.0$ & fitted momentum in MeV \\
\hline fitpar.td $<1.0$ & tangent of the track dip angle \\
\hline fitpar.td $>0.577$ & same as above \\
\hline t0 $>710$ & time in nsec relative to center of proton pulse \\
\hline nactive $\geq 20$ & number of drift tubes used in fit \\
\hline t0err $<1.5$ & fit momentum uncertainty \\
\hline fitmomerr $<0.2$ & $\chi^{2}$ fit confidence level \\
\hline fitcon $>0.0001$ & distance of closest approach to longitudinal axis \\
\hline Abs(fitpar.d0) $<100$ &
\end{tabular}

We then adopted a more sophisticated technique, using the Figure of Merit (FM) tool. The FM tool is an application that performs sensitivity optimization analysis in the situation of rare-event signal and background files. The FM tool is described in detail in Reference [23]. The FM tool works with momentum spectra, performs cut optimizations (which are optimized to a defined figure of merit), and computes the sensitivity associated with an experiment which had delivered those signal and background momentum spectra. 
The purpose of the FM tool is to take track reconstruction root files containing the electron track information (produced in the Mu2e Offline simulation) for signal and related background containing the electron track properties and produce a "Figure of Merit" related to the Feldman-Cousins 90\% CL sensitivity [24]. The FM tool takes momentum spectra of both signal and background and "smears" them with a function that represents the detector's expected resolution. The FM tool then applies minimum quality cuts on track information and performs optimization on the momentum signal window to minimize background and maximize signal events. Following this, the FM tool will produce a 90\% CL sensitivity based on the momentum spectra provided. The FM tool currently handles three types of data files:

- Conversion Signal files, containing electron tracks from conversion electrons

- DIO background files containing electron tracks from decay in orbit electrons

- RPC background files containing electron tracks produced via radiative pion capture

In this study only CE signal and DIO background files are considered. 


\section{CHAPTER 5}

\section{VARIATIONS OF THE TARGET CONFIGURATIONS}

Variations on the muon stopping target configuration are conducted to determine what effects several geometric aspect of the target system have on the signal efficiency and what the optimal configuration is for the best overall sensitivity. Beginning with the standard 17-layer configuration, variations on radius, thickness, spacing, and mass are investigated. It is possible that some conversion electrons will interact with the proton absorber and lose energy. At the time of the study there was no confirmed geometry of the proton absorber and therefore it was removed from the simulation so to isolate any effect in signal acceptance to be from changes to the stopping target. To account for this, an additional cut of momentum greater than $104.0 \mathrm{MeV} / \mathrm{c}($ fitmom > 104.0 $)$ was added to list of standard track cuts in Table 4.1.

Several constraints are placed on the variations, including keeping mass equal to the standard target system and keeping all layers within the standard target system longitudinal length. The mass constraint allows us to compare systems equally, as background rates increase with mass. Keeping the system in the standard target length is due to effects of the magnetic field, and being outside the constant magnetic field region can have detrimental effects on track reconstruction. The CE yield and FM tool 90\% CL sensitivity are shown for each configuration. Error bars are shown on all yield plots. A test was conducted on the variation of the 90\% CL sensitivity due to different starting seeds. A total of 15 simulations of one configuration with different starting random seeds for both muons stopping and 
electron track reconstruction showed the variation in the $90 \%$ CL sensitivity to be $\pm 0.01 \times 10^{-16}$.

\subsection{Increasing number of target layers}

The first study conducted was to investigate what effect the total number of target layers and layer thickness have on signal efficiency. The motivation for decreasing the layer thickness is that thinner layers would result in less energy loss for conversion electrons with high $\mathrm{p}_{T}$. High $\mathrm{p}_{T}$ electrons will lose energy as they pass through the layer they were born in and adjacent layers; therefore reducing the thickness will reduce energy loss.

Several geometric conditions were required for this study. The total mass of the targets was kept equal to the standard 17-layer target system. The position and length of the target system was also kept equal to the original system. The slope of the taper of layer radius was kept equal to the standard 17-layer configuration, meaning the farthest upstream layer has a radius of $83 \mathrm{~mm}$ and the farthest downstream layer has a radius of $65.3 \mathrm{~mm}$ with a linear decrease in radius for layers between.

The number of layers was increased from 13 foils to 90 foils. In order to maintain these conditions the layer thickness was decreased from the standard $0.2 \mathrm{~mm}$; therefore as the number of layers increases the layer thickness decreases. The spacing between layers was also decreased as the number of layers increased. Each configuration's layer thickness and spacing is dependent on the total number of layers. In Figure 5.1 and Figure 5.2 the layer thickness and spacing is shown for two cases. 
Figure 5.3 shows that as the number of foils increases, the total yield of conversion electrons increases. This can be attributed to the layers being thinner for increasing number of foils, resulting in less energy loss. Beyond 49 layers, the increase in yield appears to be minimal. In Figure 5.4, the 90\% CL sensitivity for all increasing foil configurations is shown. The 90\% CL sensitivity decreases with increasing number of layers.

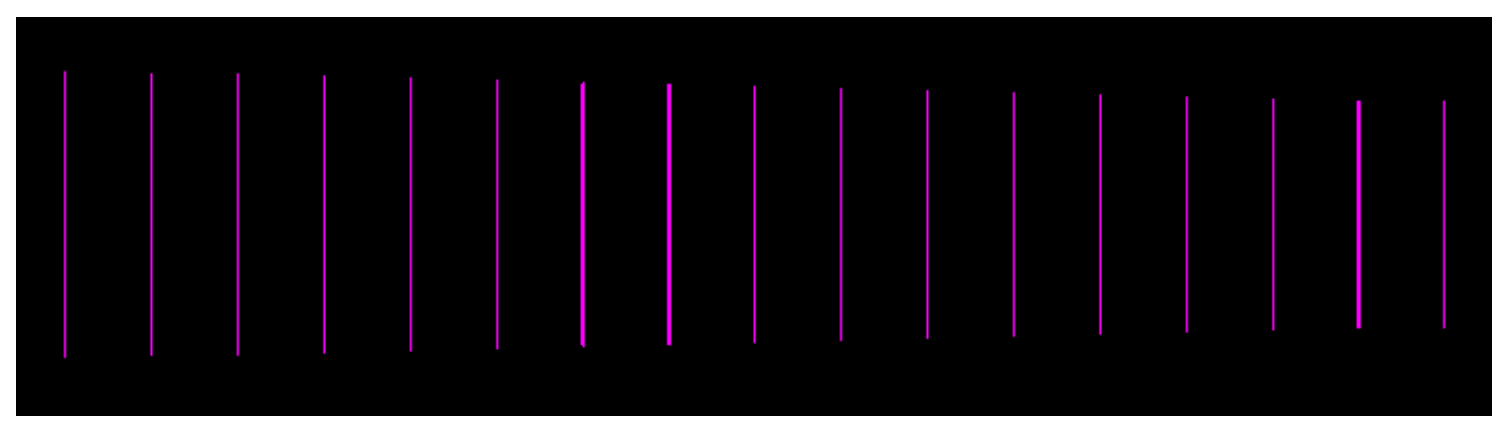

Figure 5.1: Standard stopping target layer configuration for 17 foils.

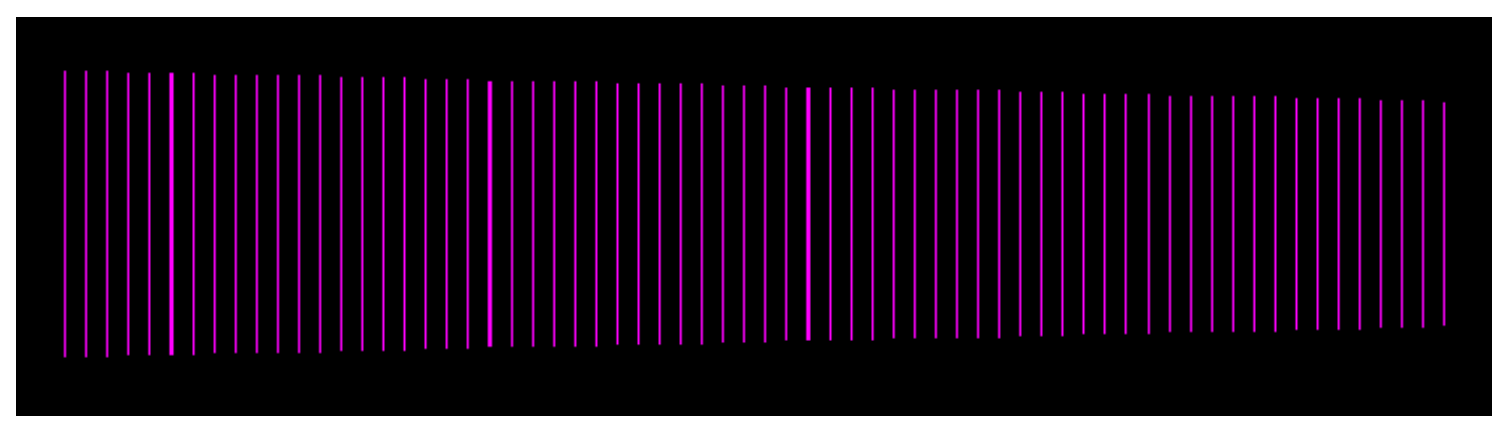

Figure 5.2: Stopping target layer configuration for 66 foils.

\subsection{The effects of layer radius tapering}

In this study, the effect the tapering of the layer radius is investigated. The motivation for downstream tapering is that conversion electrons that are pitched 


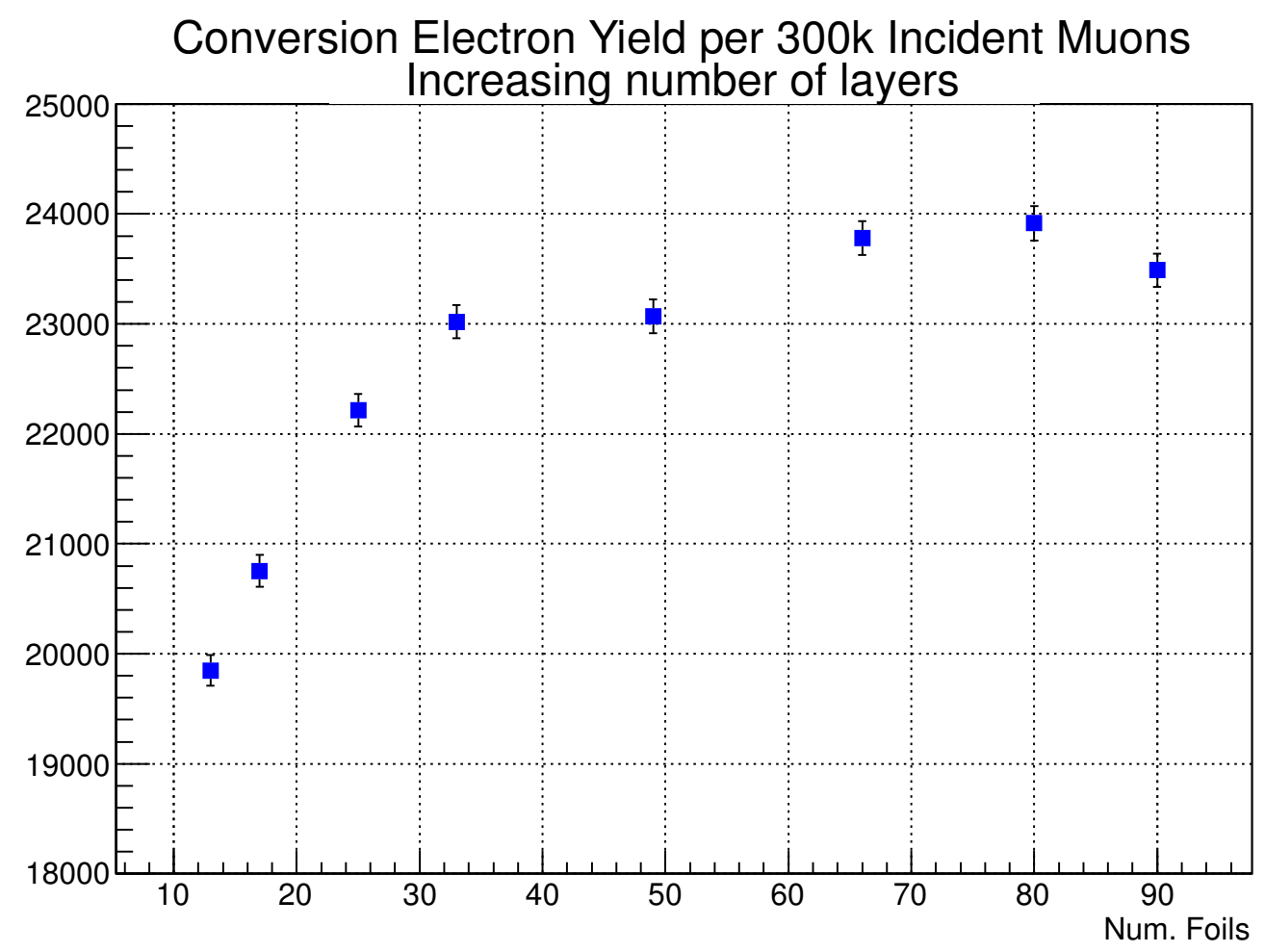

Figure 5.3: Yield versus Number of target layers from 13 to 90. 


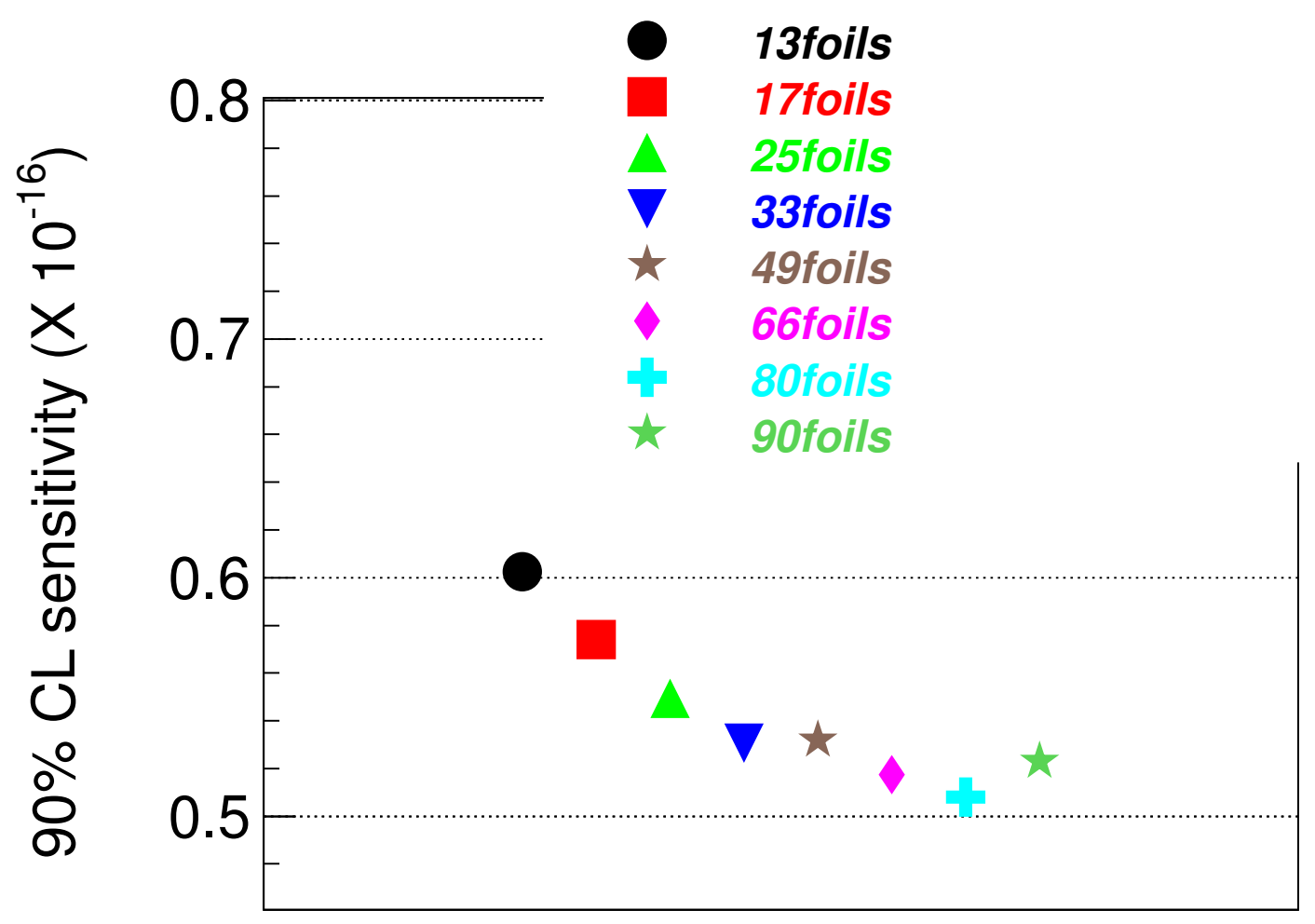

Figure 5.4: 90\% CL Sensitivity for increasing number of target layers. 
downstream toward the tracker are less likely to intersect subsequent layers of the stopping target, and therefore reduce energy loss. The motivation for upstream tapering is that equally likely conversion electrons that are pitched upstream will travel some distance before being reflected downstream (by the magnetic field) toward the tracker. While traveling upstream, these conversion electrons are less likely to intersect subsequent layers of the stopping target, and therefore reduce energy loss.

The 66-layer configuration from the previous study was chosen as the base configuration and variations were applied to this geometry. The 66-layer configuration was chosen because it was in the plateau region of Figure 5.3 and also has the best 90\% CL sensitivity. As in the previous section, several conditions were required of the geometry. The total mass of the targets was kept equal to the standard seventeen-layer target system. The position and length of the target system was also kept equal to the original system. The slope of the layer radius taper was then adjusted from nearly zero taper to a relatively large taper.

In this study both upstream and downstream tapering are considered. Downstream tapering corresponds to the largest foil radius being the farthest upstream layer and tapering to the smallest foil radius being the last foil farthest downstream (Table 5.1). Upstream tapering corresponds to the smallest foil radius being the farthest upstream and the largest foil radius being the farthest downstream (Table 5.2). Figure 5.5 and Figure 5.6 show the differences between downstream and upstream tapering of layer radii. The layer thickness was equal to the 66-layer case in the first study. The spacing between layers was also equal to the 66-layer configuration. Layer radii are changed from the original 66-layer configuration.

Figure 5.7 shows yield in conversion electrons for the downstream tapering configuration. Figure 5.8 shows that yield in conversion electrons for the upstream 
tapering configuration. There are minimal differences between configurations, suggesting the degree of tapering in either the downstream or upstream direction has no effect. In Figure 5.9, the sensitivity of all downstream tapering configurations is shown. In Figure 5.10, the sensitivity of all upstream tapering configurations is shown. Again, there is minimal difference between different degrees of tapering within each configuration, which suggests that the tapering has no effect on the $90 \%$ CL sensitivity.

Table 5.1: Radius of first and last foil for downstream tapering in $\mathrm{mm}$

\begin{tabular}{|c|c|}
\hline First Radius & Last Radius \\
\hline 75.00 & 73.65 \\
\hline 80.00 & 68.50 \\
\hline 85.00 & 63.11 \\
\hline 90.00 & 57.46 \\
\hline 95.00 & 51.52 \\
\hline
\end{tabular}

Table 5.2: Radius of first and last foil for upstream tapering in $\mathrm{mm}$

\begin{tabular}{|c|c|}
\hline First Radius & Last Radius \\
\hline 73.65 & 75.00 \\
\hline 68.50 & 80.00 \\
\hline 63.11 & 85.00 \\
\hline 57.46 & 90.00 \\
\hline 51.52 & 95.00 \\
\hline
\end{tabular}

\subsection{Symmetric tapering}

In this study, all configurations have a total of 65 layers and the effect of symmetric tapering is investigated. The choice of 65 layers was for symmetry of the 


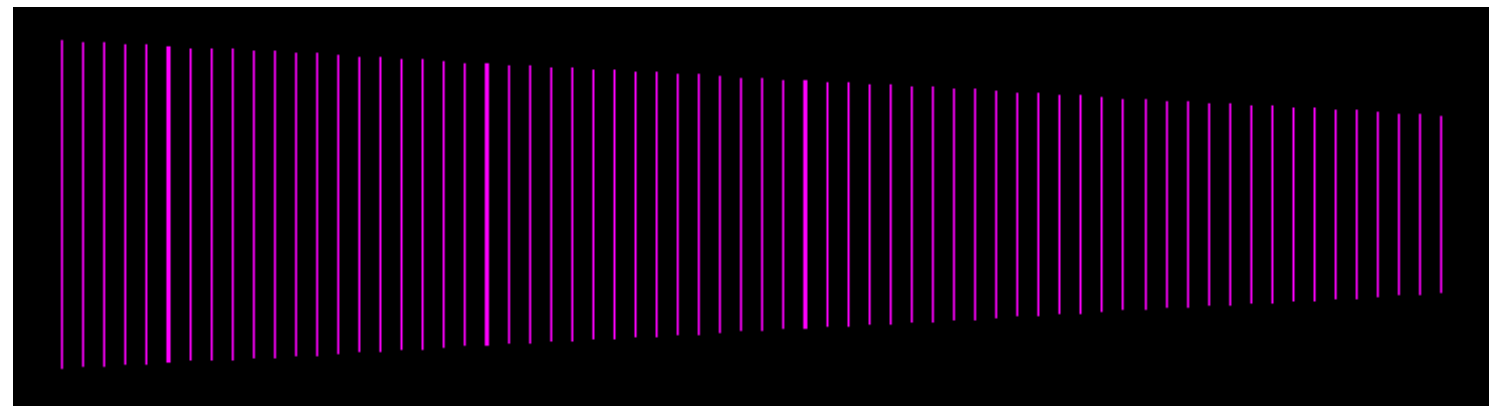

Figure 5.5: Stopping target layer configuration for 66 foils with downstream tapering (Incoming muon beam from left to right).

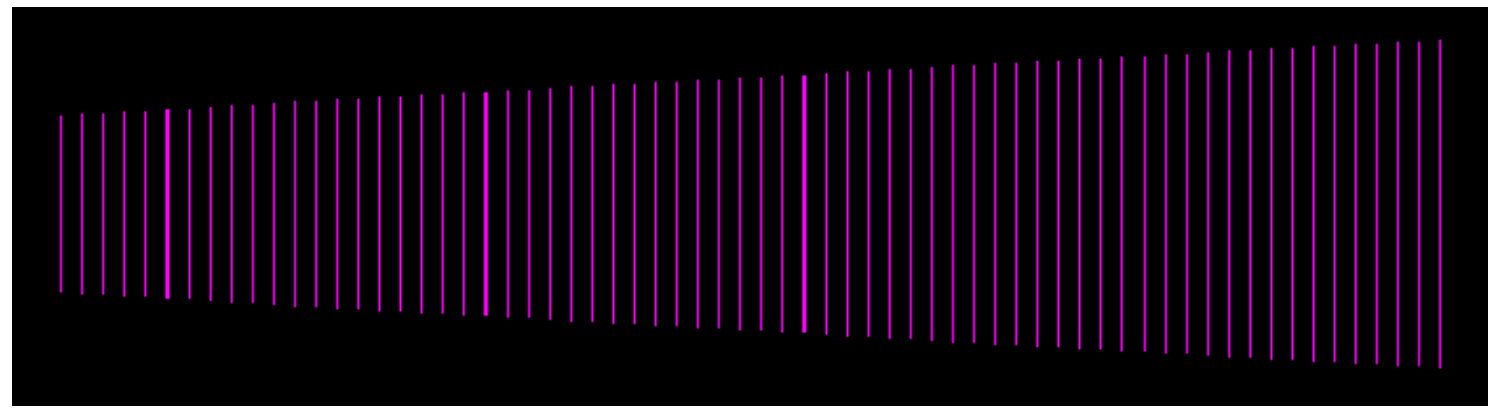

Figure 5.6: Stopping target layer configuration for 66 foils with upstream tapering (Incoming muon beam from left to right). 


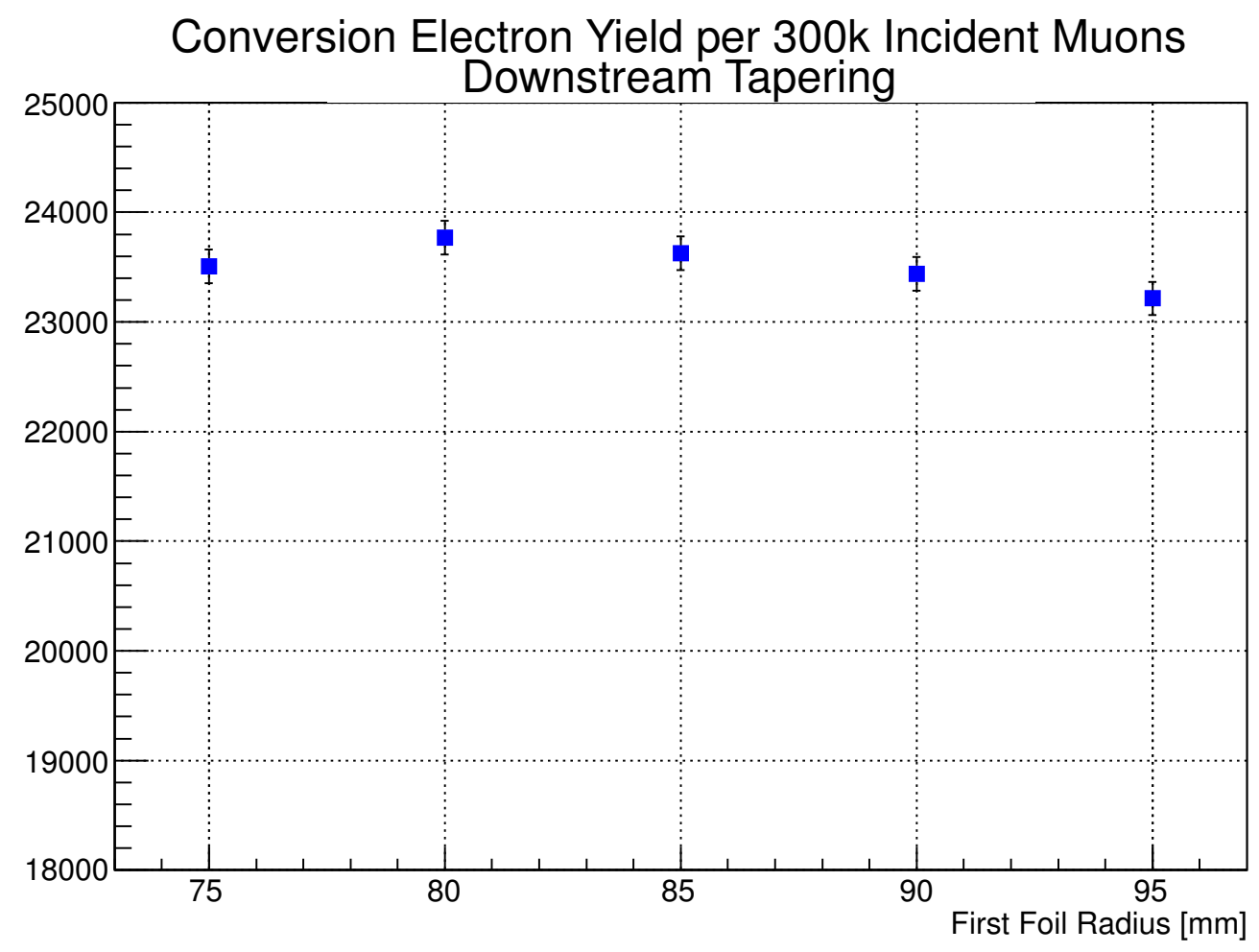

Figure 5.7: Yield versus foil radius for downstream tapering. 


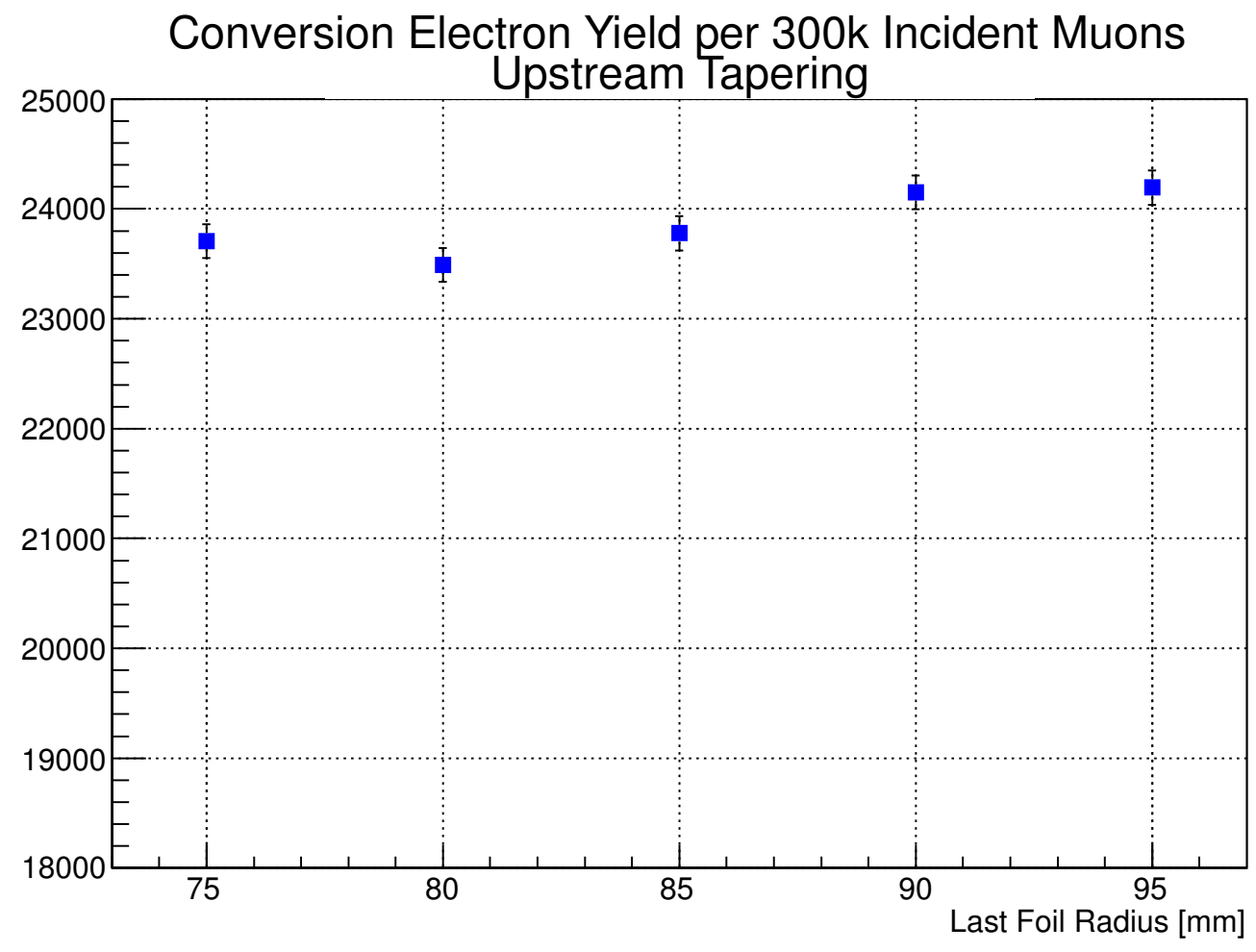

Figure 5.8: Yield versus foil radius for upstream tapering. 


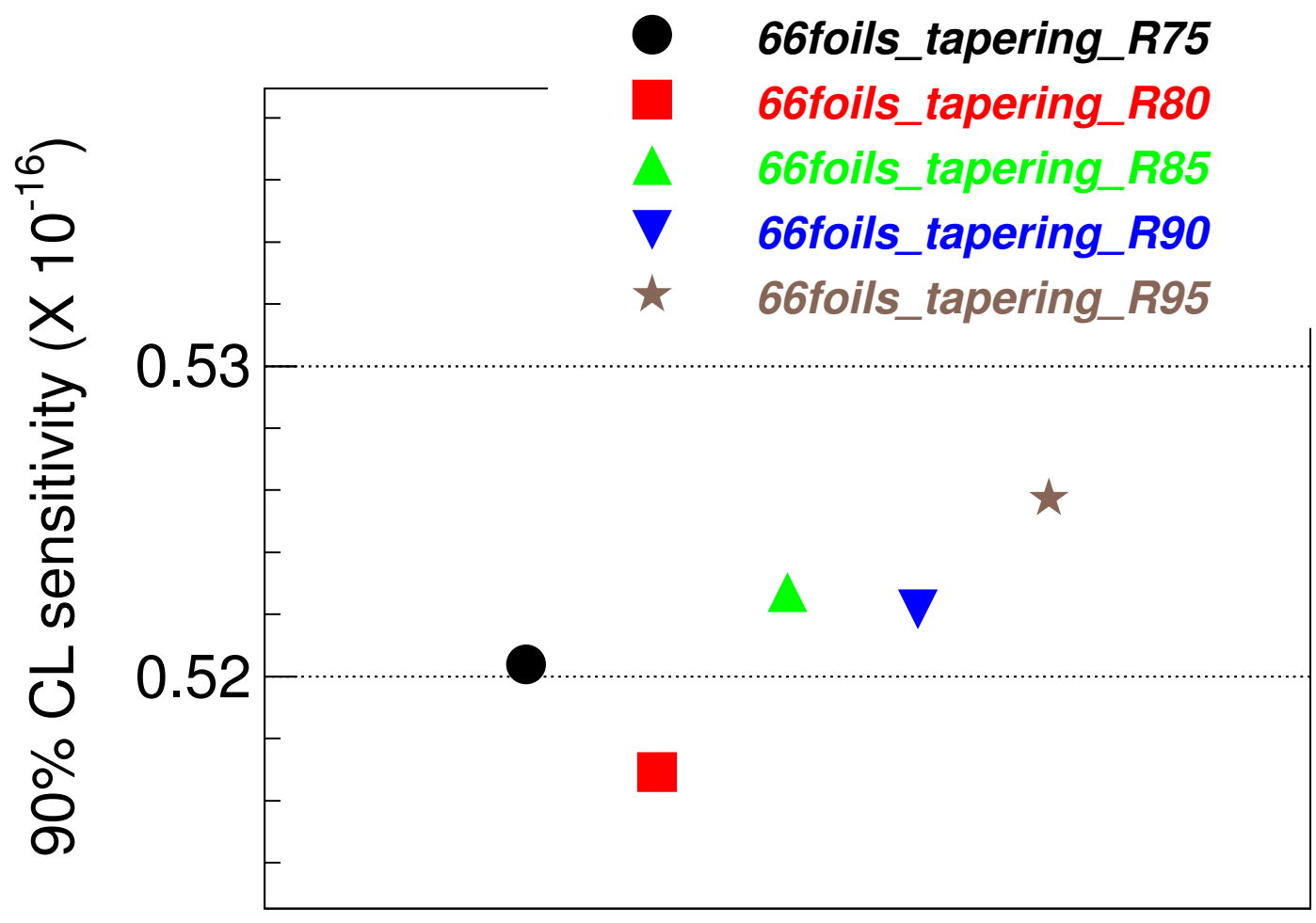

Figure 5.9: 90\%CL sensitivity for all configuration of downstream tapering of target layer radius. R75 corresponds to the largest (first) foil radius of $75 \mathrm{~mm}$. 


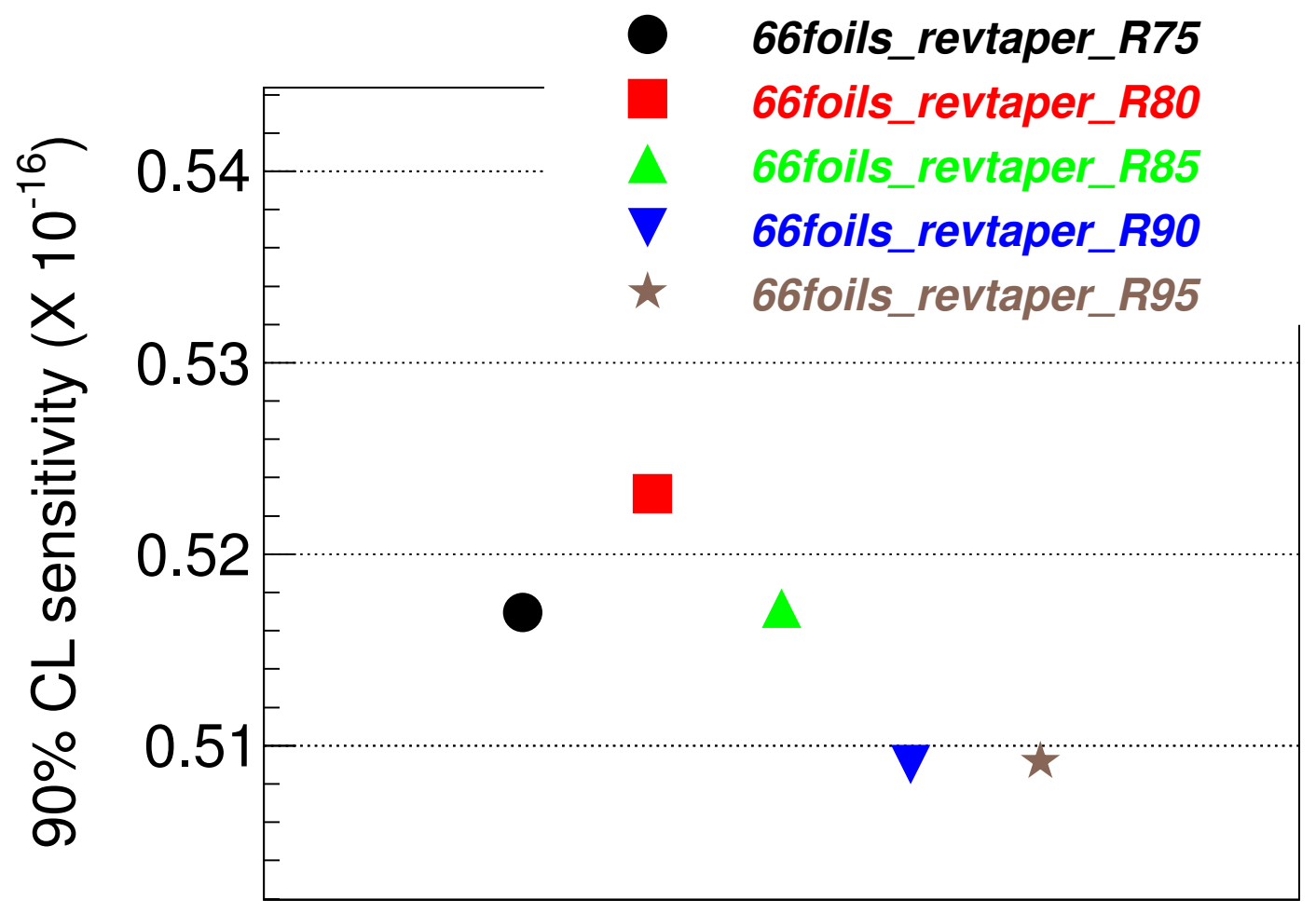

Figure 5.10: 90\%CL sensitivity for all configuration of upstream tapering of target layer radius. R75 corresponds to the largest (last) foil radius of $75 \mathrm{~mm}$. 
target, such that a single central layer has the largest radius. The configuration consists of two sections of layers with radius tapering in opposite directions (upstream and downstream). The motivation for symmetric tapering is that conversion electrons that are either pitched downstream or upstream are less likely to intersect subsequent layers of the stopping target, and therefore reduce energy loss.

As in the previous studies, several conditions were required of the geometry. The total mass of the target system was kept equal to the standard seventeen-layer target system. The position and length of the target system was also kept equal to the original system.

At the farthest upstream end of the target system, the layers have the smallest radius. The radii of layers increases linearly to the central foil with maximum radius, and then decreases linearly to the farthest downstream foil. Figure 5.11 shows the symmetric tapering configuration with central foil radius and layer spacing. The central foil radius is increased, beginning at $75 \mathrm{~mm}$ up to $95 \mathrm{~mm}$ in $5 \mathrm{~mm}$ increments. The spacing between layers was increased slightly from the 66-layer configuration in the first study due to having 65 layers.

Figure 5.12 shows conversion electron yield for all symmetric tapering configurations. There is minimal difference in yield for all configurations, suggesting that symmetric taper has no effect. In Figure 5.13, 90\% CL sensitivity of all symmetric tapering configurations is shown. There are minimal differences in 90\% CL sensitivity for all symmetric tapering configurations. 


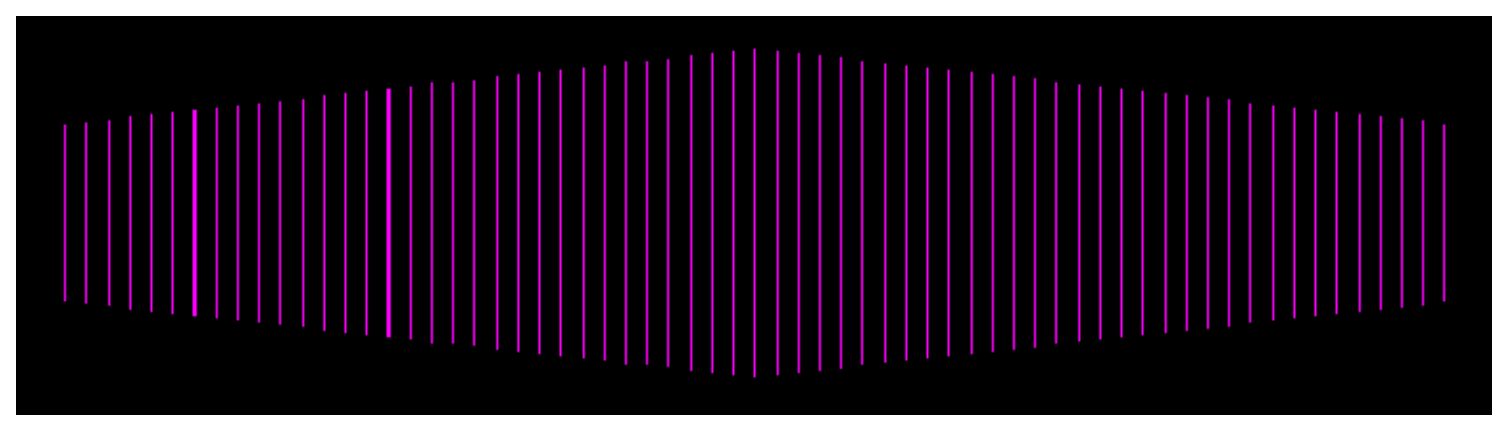

Figure 5.11: Stopping target layer configuration for 65 foils with symmetric tapering (Incoming muon beam from left to right).

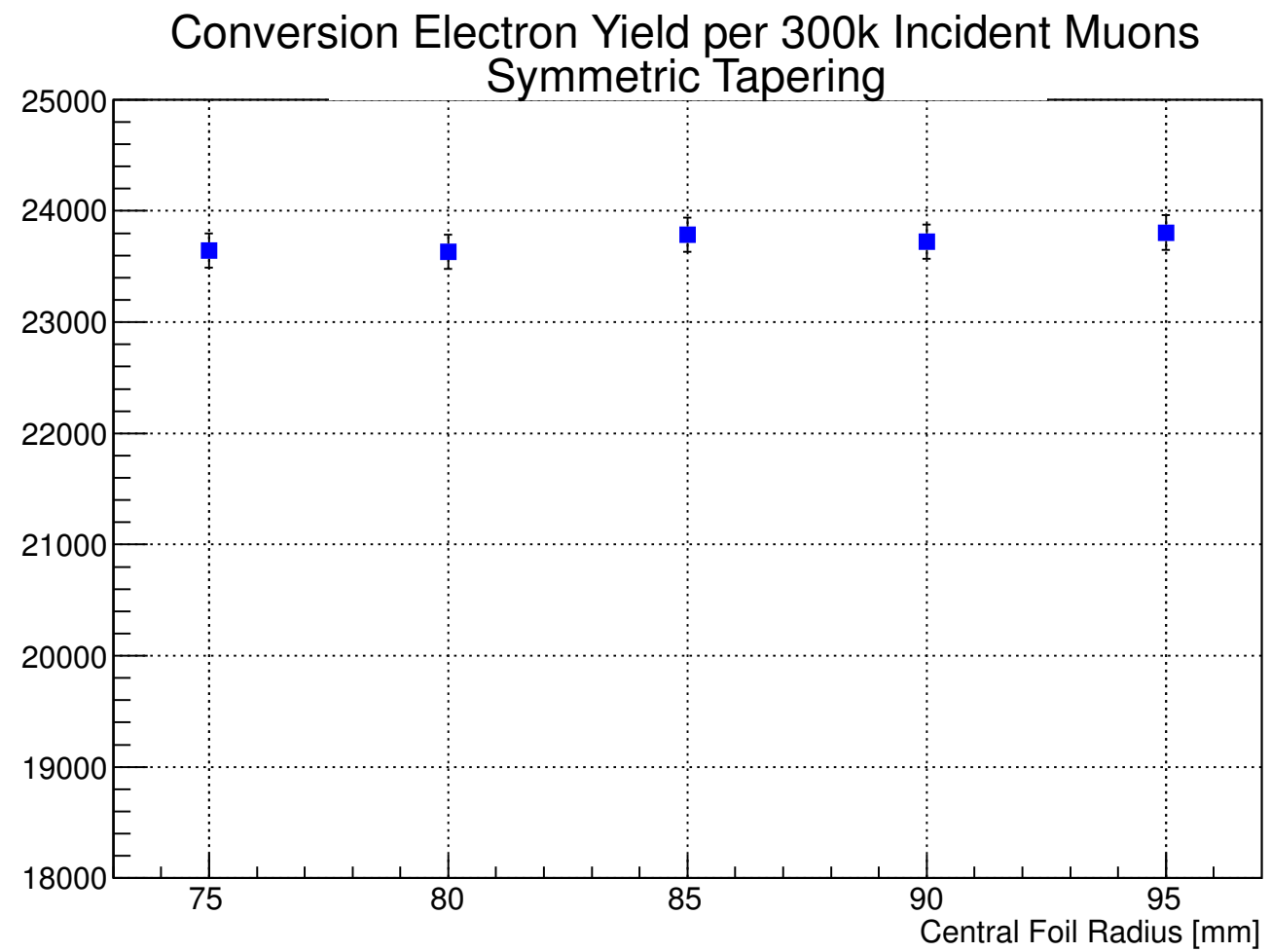

Figure 5.12: Yield versus foil radius for symmetric tapering. 


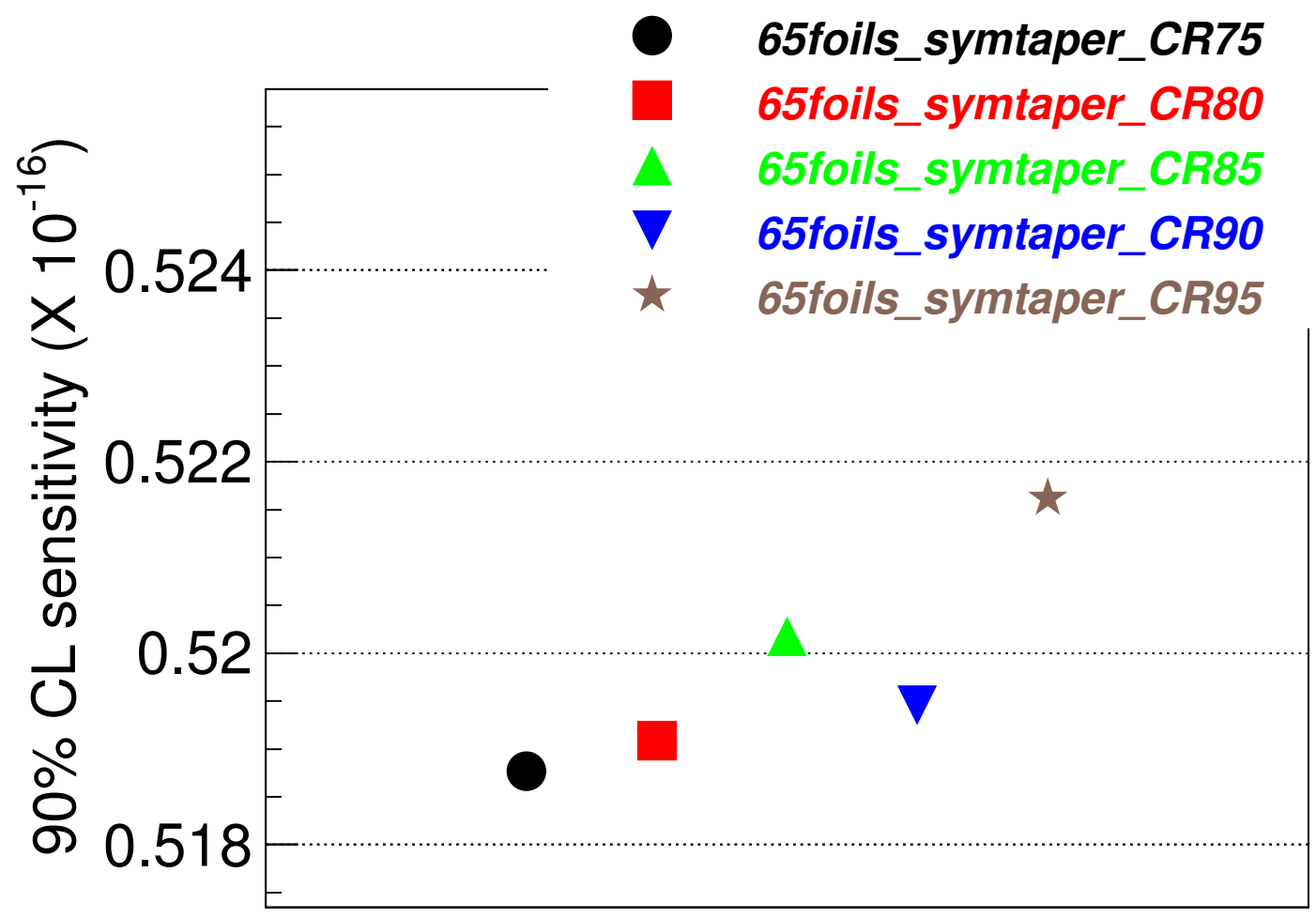

Figure 5.13: 90\% CL Sensitivity for all configuration of symmetric tapering of target layers. CR75 corresponds to the central foil having a radius of $75 \mathrm{~mm}$. 


\subsection{Constant radius with decreasing thickness}

In this study, all configurations have a total of 66 layers and the effect of larger radius and reduced thickness is investigated. All layers have an equal radius; therefore there is no tapering of layer radii. As in the previous, several conditions were required of the geometry. The total mass of the target system was kept equal to the standard seventeen-layer target system. The position and length of the target system was also kept equal to the original system.

The layer radius for all layers is increased, beginning at $75 \mathrm{~mm}$ up to $95 \mathrm{~mm}$ in

$5 \mathrm{~mm}$ increments. In order to maintain the geometric requirements, as the radius of layers increases, the layer thickness decreases. The spacing between layers was also equal to the 66-layer configuration in the first study. Figure 5.14 shows the constant radius configuration and layer spacing.

Figure 5.15 shows conversion electron yield for all constant radius - constant mass configurations. There is approximately equal yield in conversion electrons at $75,80,85 \mathrm{~mm}$ radii and this yield is slightly higher than the yield at 90 and $95 \mathrm{~mm}$ radii. In Figure 5.16, 90\% CL sensitivity for all constant radius - constant mass configurations is shown. There are minimal differences in 90\% CL sensitivity for all constant radius with decreasing thickness (constant mass) configurations.

\subsection{Constant radius with constant thickness}

In this study, all configurations have a total of 66 layers and the effect of larger radius and increased mass is investigated. All layers have an equal radius; therefore there is no tapering of layer radii. The position and length of the target system 


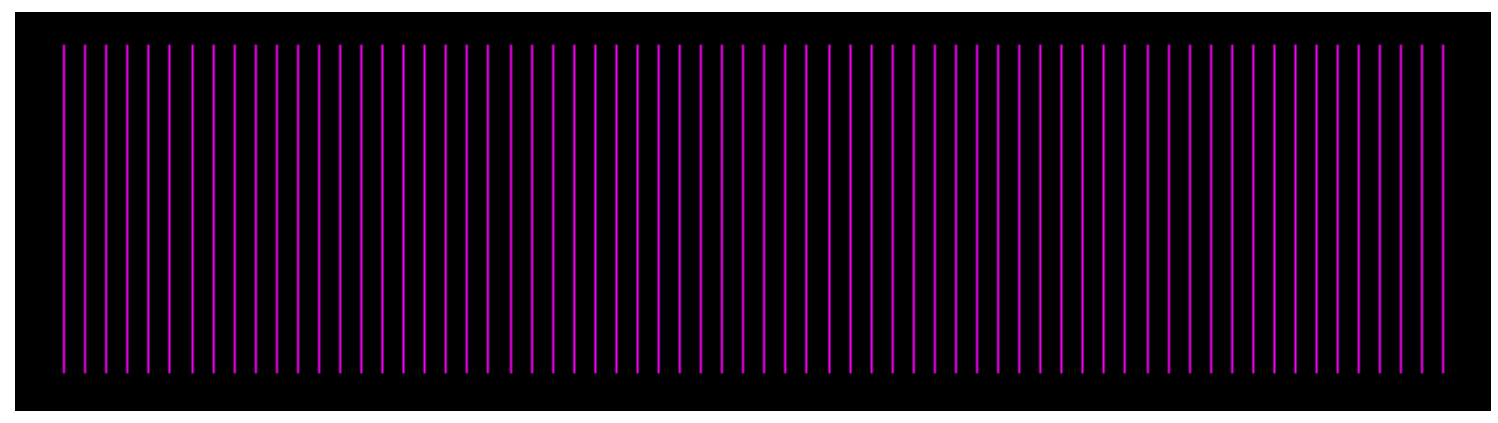

Figure 5.14: Stopping target layer configuration for 66 foils with constant radius (Incoming muon beam from left to right).

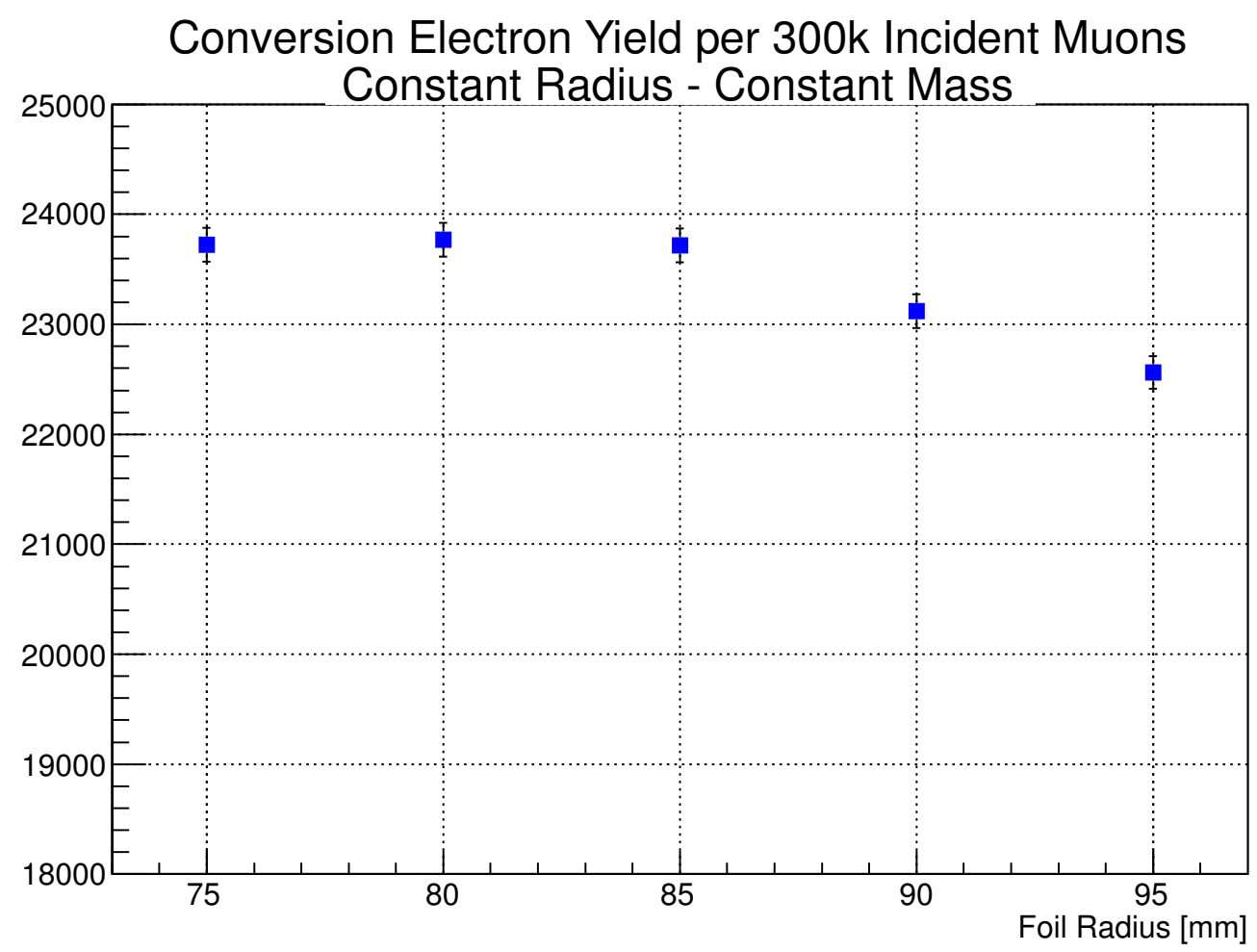

Figure 5.15: Yield versus foil radius for constant radius and constant mass. 


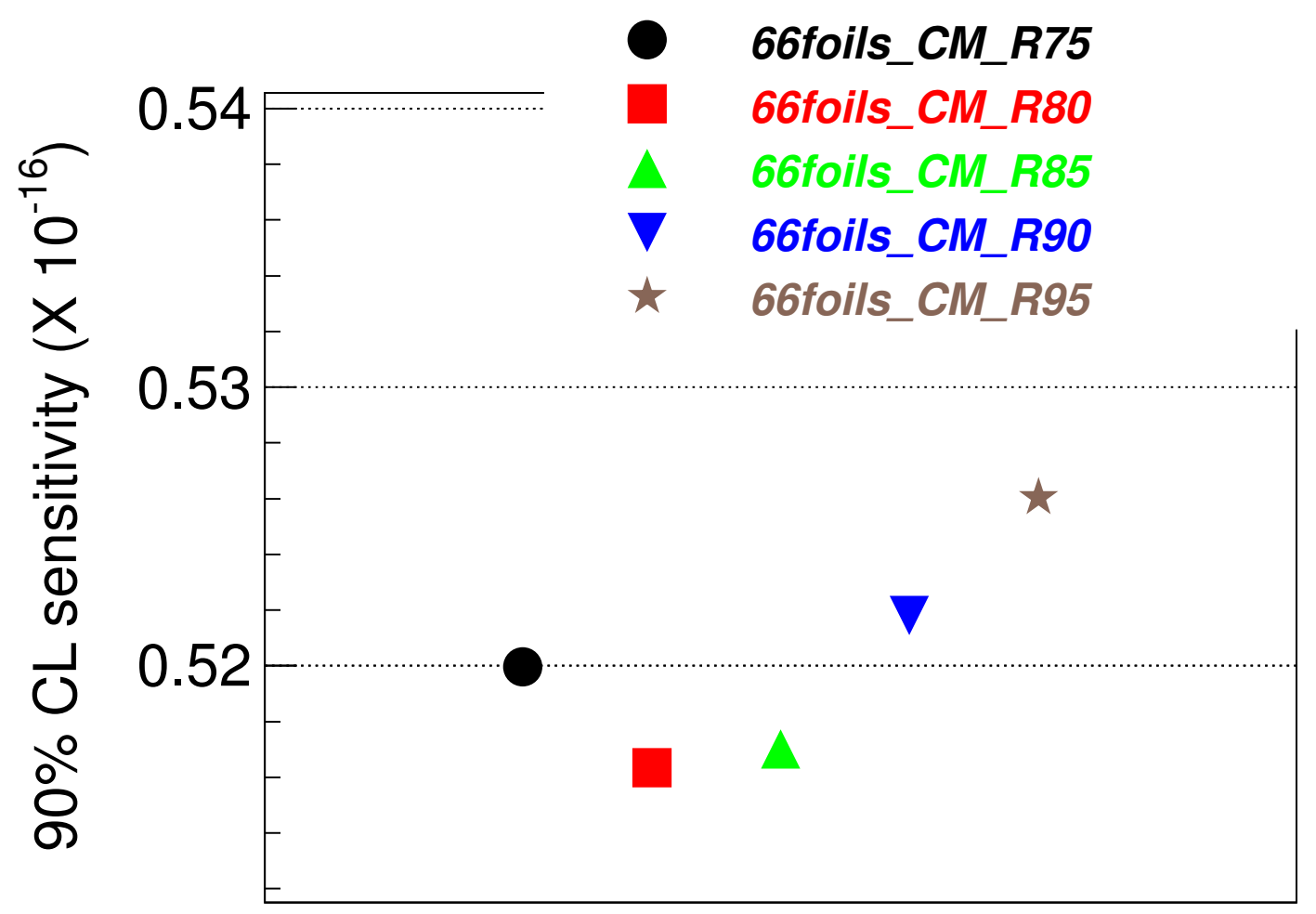

Figure 5.16: 90\% CL sensitivity for all configuration of constant mass and increasing layer radius (decreasing thickness). R75 corresponds to the radius of $75 \mathrm{~mm}$ for all foils. 
was kept equal to the original system. However, the total mass of the targets was allowed to vary from the standard seventeen-layer target system.

The layer radius for all layers is increased, beginning at $75 \mathrm{~mm}$ up to $95 \mathrm{~mm}$ in $5 \mathrm{~mm}$ increments. Since the constant mass requirement is not enforced, all configurations have the same layer thickness. Therefore as the radius increases the total mass of the target system will increase. The spacing between layers was also equal to the 66-layer configuration in the first study.

Figure 5.17 shows conversion electron yield for all constant radius - constant thickness (increasing mass) configurations. There is a slight increase in yield for $85 \mathrm{~mm}$ radius, but a minimal effect overall. In Figure 5.18, 90\% CL sensitivity of all constant radius - constant thickness configurations is shown. There is slight improvement in the $90 \%$ CL sensitivity between the $75 \mathrm{~mm}$ radius and $95 \mathrm{~mm}$ radius constant thickness configurations. This can be attributed to the increased number of conversion electrons due to a higher target mass. 


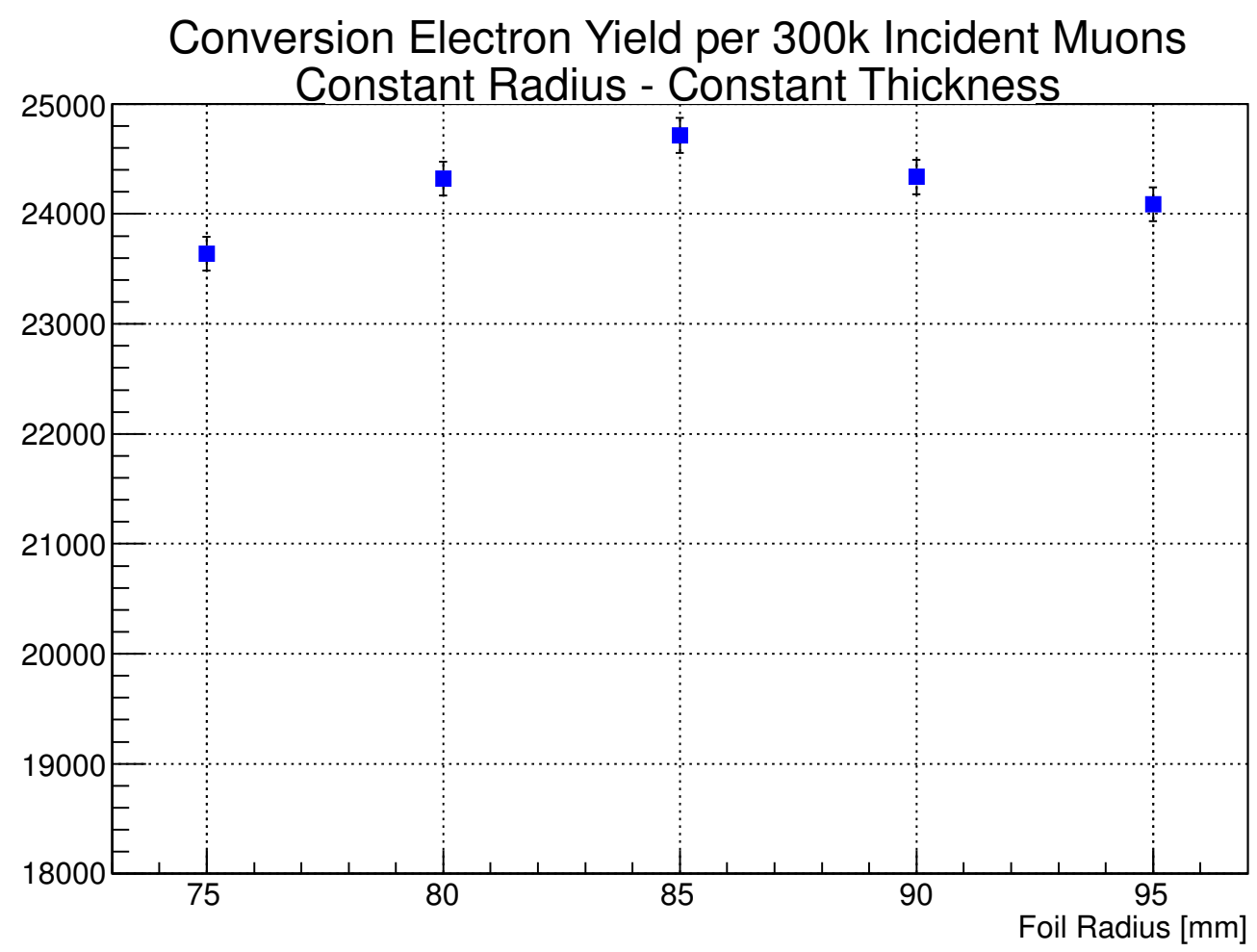

Figure 5.17: Yield versus foil radius for constant radius and constant thickness. 


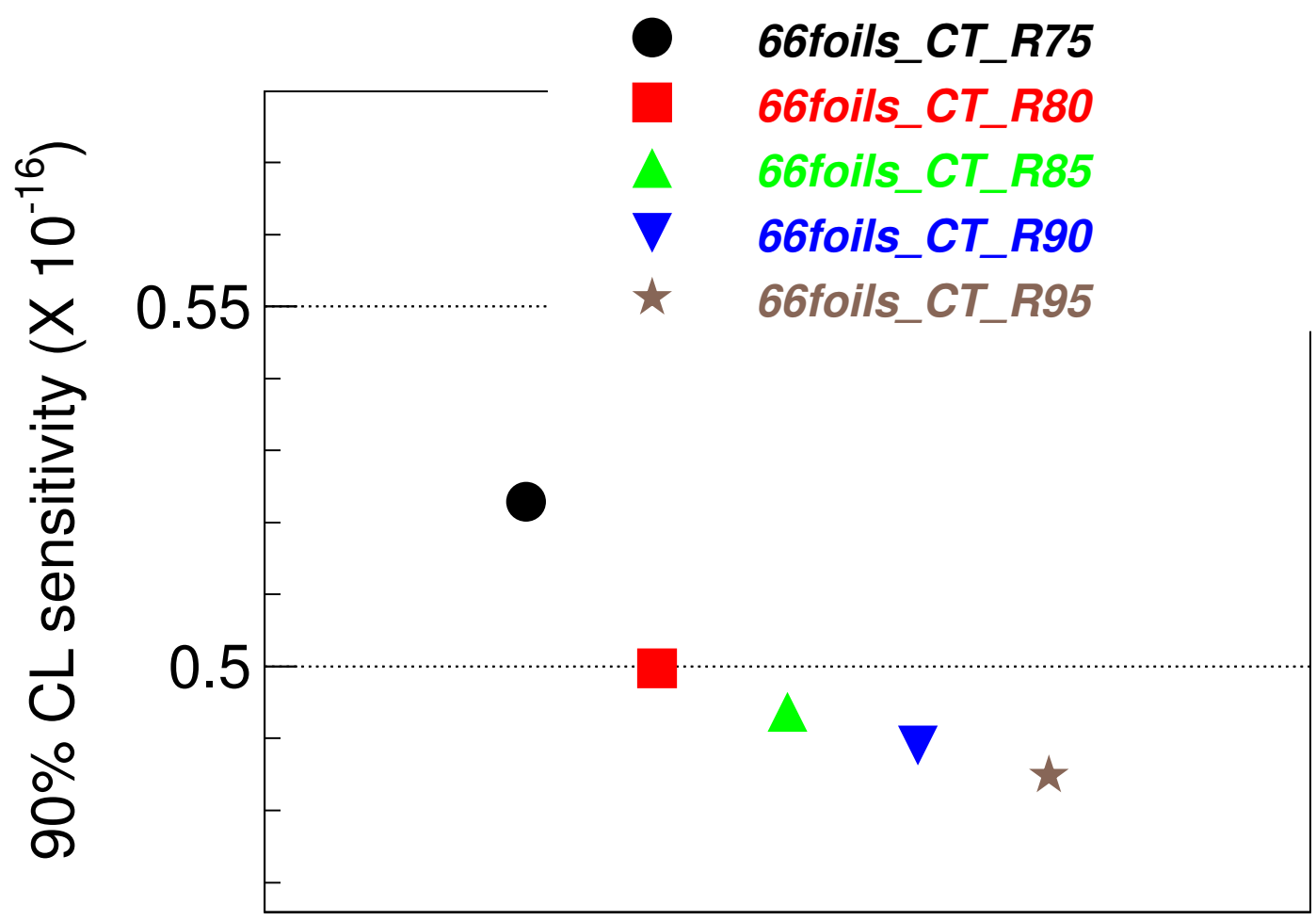

Figure 5.18: 90\% CL Sensitivity for all configuration of constant thickness and increasing layer radius (increasing mass). R75 corresponds to the radius of $75 \mathrm{~mm}$ for all foils. 


\section{CHAPTER 6 CONCLUSION}

This thesis represents a first step in full optimization of the muon stopping target using the Mu2e Offline. Also, the FM tool has proven to be a powerful instrument in determining what effects changes to the physical geometry have on the overall sensitivity of the Mu2e Experiment.

Since the mass, thickness, and radius are intrinsically linked, determining what aspect of the geometry has the greatest effect on the sensitivity of the experiment is difficult. These studies have shown that the tapering of the foil radii has a negligible effect on the $90 \%$ CL sensitivity and that increasing the number of layers or decreasing the thickness of layers leads to improvements in the 90\% CL sensitivity. Also, increasing the total mass of the target by holding the thickness constant and increasing the radius leads to an improvement of the $90 \%$ CL sensitivity.

The configurations presented in this thesis immediately show a possible significant gain in the sensitivity of the experiment. The target configuration with constant thickness and constant radius of $95 \mathrm{~mm}$ (increased mass) performed the best out of all configurations, with a $90 \%$ CL sensitivity of $0.465 \times 10^{-16}$. This is an $18 \%$ improvement over the standard stopping target configuration. Further studies of other

geometries and configurations must be conducted, as well as a full optimization of whatever chosen geometry. Also, the inclusion of RPC backgrounds are necessary before any final target configuration can be chosen. 


\section{REFERENCES}

[1] J. L. Hewett et al., Fundamental Physics at the Intensity Frontier, 2012, arxiv:1205.2671v1[hep-ex].

[2] D. J. Griffiths, Introduction to Elementary Particles, John Wiley \& Sons, Inc., 1987.

[3] S. L. Glashow, J. Iliopoulos, and L. Maiani, Phys. Rev. D 2, 1285 (1970).

[4] Y. Fukuda et al. (Super-Kamiokande Collaboration), Evidence for oscillation of atmospheric neutrinos, 1998, arXiv:hep-ex/9807003.

[5] W. J. Marciano, T. Mori, and J. M. Roney, Annu. Rev. of Nucl. Part. Sci 58, 315 (2008).

[6] S. Ahmad et al., Phys. Rev. D 38, 2102 (1988).

[7] The Mu2e Collaboration, Proposal to search for $\mu^{-} \mathrm{N} \rightarrow e^{-} \mathrm{N}$ with a Single Event Sensitivity Below 10 ${ }^{-16}$, 2008, mu2e-doc-388-v1.

[8] The MEG Collaboration, Search for $\mu^{+} \rightarrow e^{+} \gamma$ down to $10^{-14}$ branching ratio, 2012, mu2e-doc-2203-v1.

[9] W. Bertl et al. (SINDRUM II Collaboration), Eur. Phys. J. C 47, 337 (2006).

[10] J. Adam et al. (MEG Collaboration), New constraint on the existence of the $\mu^{+} \rightarrow e^{+} \gamma$ decay, 2013, arxiv:1303.0754v2[hep-ex].

[11] M. L. Brooks et al. (MEGA Collaboration), Phys. Rev. Lett. 83, 1512 (1999).

[12] K. Abe et al. (Belle Collaboration), A New Search for tau $\rightarrow$ mu gamma and tau $\rightarrow$ e gamma Decays at Belle, 2006, arXiv:hep-ex/0609049.

[13] B. Aubert et al. (Babar Collaboration), Searches for Lepton Flavor Violation in the Decays tau $\rightarrow$ e gamma and tau $\rightarrow$ mu gamma, 2009, arXiv:0908.2381v2 [hep-ex].

[14] The Mu2e Collaboration, Mu2e Conceptual Design Report, 2012, mu2e-doc1169-v21.

[15] R. Bernstein, D. Brown, and J. Miller, Summary of Signal and Background Estimates for CDR, 2012, mu2e-docdb-2085. 
[16] A. Czarnecki, X. G. i Tormo, and W. J. Marciano, Muon Decay in orbit: spectrum of high-energy electrons, 2011, arxiv:1106.4756v2[hep-ph].

[17] J. A. Bistirlich, K. M. Crowe, A. S. L. Parsons, P. Skarek, and P. Truöl, Phys. Rev. C 5, 1867 (1972).

[18] D. Measday, Physics Reports 354, 243 (2001).

[19] R. Kutschke, Units and Coordinate Systems Used by Mu2e Offline Software, 2010, mu2e-doc-1120-v1.

[20] J. Beringer et al., Phys. Rev. D 86, 010001 (2012).

[21] S. Agostinelli et al., Nuclear Instruments and Methods in Physics Research Section A: Accelerators, Spectrometers, Detectors and Associated Equipment 506, 250 (2003).

[22] Particle Data Group, Atomic and nuclear properties of materials: Aluminum (Al), 2012, http://pdg.lbl.gov/2012/AtomicNuclearProperties/ MUON_ELOSS_TABLES/muonloss_013.dat.

[23] M. Fischler, A User's Guide for the Mu2e Figure of Merit Tool FofM, 2012, mu2e-doc-2227-v1.

[24] G. J. Feldman and R. D. Cousins, A Unified Approach to the Classical Statistical Analysis of Small Signals, 1999, arXiv:physics/9711021 [physics.data-an].

[25] R. Bernstein, Variations of Stopping Target Configuration, 2011, mu2e-doc1857-v3.

[26] S. Albino, F. Deppisch, and R. Ruckl, Supersymmetric Lepton Flavor Violation and Leptogenesis, 2006, arXiv:hep-ph/0606226.

[27] The COMET Collaboration, An Experimental Search for Lepton Flavor Violating $\mu^{-}-e^{-}$Conversion at Sensitivity of $10^{-16}$ with a Slow-Extracted Bunched Proton Beam, 2012, mu2e-doc-2202-v2.

[28] The MECO Collaboration, Search for $\mu \mathrm{N} \rightarrow \mathrm{e} N$ with Sensitivity Below $10^{-16}$, 2008, mu2e-doc-378-v1.

[29] C. Ozben, Y. Semertzidis, and B. Morse, Optimization of the Muon Stopping Target of MECO, 2010, mu2e-doc-762-v1.

[30] D. Brown, E. Charles, and D. Roberts, The BaBar Track Fitting Algorithm, 2010, mu2e-doc-781-v1. 